\title{
Computation of Al-Salam Carlitz and Askey-Wilson moments using Motzkin paths
}

\author{
Gaspard Ohlmann \\ Department of Partial differential equations \\ EPFL, Ecole Polytechnique Federale de Lausanne \\ Switzerland \\ gaspard.ohlmann@unibas.ch
}

Submitted: Aug 13, 2020; Accepted: May 13, 2021; Published: Jul 2, 2021

(C) The author. Released under the CC BY-ND license (International 4.0).

\begin{abstract}
In this paper we study the moments of polynomials from the Askey scheme, and we focus on Askey-Wilson polynomials. More precisely, we give a combinatorial proof for the case where $d=0$. Their values have already been computed by Kim and Stanton in 2015, however, the proof is not completely combinatorial, which means that an explicit bijection has not been exhibited yet. In this work, we use a new combinatorial approach for the simpler case of Al-Salam-Carlitz, using a sign reversing involution that directly operates on Motzkin path. We then generalize this method to Askey-Wilson polynomials with $d=0$ only, providing the first fully combinatorial proof for that case.
\end{abstract}

Mathematics Subject Classifications: 05A10, 33D50,05A19

\section{Introduction}

Enumeration of objects is a powerful tool for understanding their properties and revealing hidden connections between them. Among other things, constructing explicit bijections is widely considered to be the best way to enumerate objects because bijections give us insight to understand how objects are related to each other. An interesting feature of combinatorics is that it is often related to other fields in mathematics. In this paper, we focus on the combinatorics of orthogonal polynomials. It is known by Favard that any monic polynomial $P_{n}(x)$ satisfies a three-term recurrence $P_{n+1}(x)=\left(x-b_{n}\right) P_{n}(x)-$ $\lambda_{n} P_{n-1}(x)$. Viennot's theory [14] provides a connection bewteen orthonal polynomials and combinatorics. Indeed, Viennot showed that the $n$th moment is the sum of weighted Motzkin paths of length $n$ whose weights are defined using $b_{n}$ and $\lambda_{n}$. Askey-Wilson polynomials (defined in [2]) moments depend of four parameters $a, b, c$ and $d$. AskeyWilson moments have been studied with Motzkin paths by Kim and Stanton [10]. The 
starcaise tableaux, introduced by Corteel and Williams in [4] and studied in [3] were also used to study those polynomials. Ismail, Stanton and Viennot [5] used $q$-Hermite Polynomials and inhomogenous matchings and Kim and Stanton [10] recently computed the general moment of Askey Wislon polynomials. However, this proof is not completely combinatorial. We compute Askey-Wilson moments using uniquely Motzkin paths, thus providing a combinatorial proof; using a sign reversing involution on Motzkin paths. A combinatorial proof has been already given for a more specific case $(c=d=0)$ in [10] using a bijection between Motzkin paths and Doubly Striped Skew Shapes and a sign reversing involution on Striped Skew Shapes. The proof we are looking for is more general (case $d=0$ ) and operates directly on Motzkin paths. In order to find a solution to the previously described problem, we also focused on a simpler family of orthogonal polynomials: the Al-Salam-Carlitz polynomials. Their moments have already been computed with a combinatorial proof using Striped Skew Shapes by Dongsu Kim. However, the Striped Skew Shapes seem to be specific to this case and it was not possible to generalize them to our situation. Looking at the effects of the sign reversing involution at the Motzkin paths level, we find patterns and behaviors that seem to reproduce in our more general case. We use this phenomenon to define a less specific sign reversing involution at the Motzkin paths level. In the first section, we look at the Al-Salam-Carlitz polynomials case, and propose a new proof for the value of their moments. This proof is rather simple, but the method and the formalism we introduce will pave the way for the last section, in which we extend the method to the Askey-Wilson polynomials moments in the case $d=0$ only.

\section{Context and goal}

In this section, we introduce the notations and some of the different approaches used for this problem ; giving a very brief review of the litterature on the subject. In particular, we define Askey-Wilson polynomials as well as their moments, and explain three approaches that have been used for computing those moments. Later, we recall the method used in [10] that makes use of Striped Skew shapes, and explain how we plan to generalize it to the case of Askey-Wilson polynomials case.

\subsection{Notation and Definition}

We use the following standard notations:

$$
\begin{gathered}
{[n]_{q}=1+q+\cdots+q^{n-1}, \quad[n]_{q} !=[1]_{q}[2]_{q} \cdots[n]_{q},} \\
{\left[\begin{array}{c}
n_{1}+\cdots+n_{k} \\
n_{1}, \ldots, n_{k}
\end{array}\right]_{q}=\frac{\left[n_{1}+\cdots+n_{k}\right]_{q} !}{\left[n_{1}\right]_{q} ! \cdots\left[n_{k}\right]_{q} !}, \quad\left[\begin{array}{c}
n \\
k
\end{array}\right]_{q}=\left[\begin{array}{c}
n \\
k, n-k
\end{array}\right]_{q},} \\
(a)_{n}=(1-a)(1-a q) \cdots\left(1-a q^{n-1}\right), \quad\left(a_{1}, \ldots, a_{k}\right)_{n}=\left(a_{1}\right)_{n} \cdots\left(a_{k}\right)_{n} .
\end{gathered}
$$

Orthogonal polynomials are a family $\left\{P_{n}(x)\right\}_{n \geqslant 0}$ of polynomials satisfying

$$
\int_{\alpha}^{\beta} P_{n}(x) P_{m}(x) w(x) d x=0 \quad \text { if } n \neq m,
$$


for constants $a, b$ and a weight function $w(x)$. The quantity

$\mu_{n}=\int_{\alpha}^{\beta} x^{n} w(x) d x$ is called the $n$th moment. Note that since the integral is linear, if we know the moments $\mu_{n}$, we can compute the integral $\int_{\alpha}^{\beta} P(x) w(x) d x$ for any polynomial $P(x)$.

The Askey-Wilson polynomials (defined in [2]) are the most general polynomials among the orthogonal polynomials classified by the Askey scheme. Special cases of the AskeyWilson polynomials include $q$-Hahn, $q$-Racah, $q$-Laguerre, $q$-Charlier, $q$-Hermite polynomials, etc.

Lastly, we introduce the unilateral basic hypergeometric serie $\phi$ :

$$
{ }_{j} \phi_{k}\left[\begin{array}{llll}
a_{1} & a_{2} & \ldots & a_{j} \\
b_{1} & b_{2} & \ldots & b_{k}
\end{array} ; q, z\right]=\sum_{n=0}^{\infty} \frac{\left(a_{1}, a_{2}, \ldots, a_{j}\right)_{n}}{\left(b_{1}, b_{2}, \ldots, b_{k}, q\right)_{n}}\left((-1)^{n} q^{\left(\begin{array}{c}
n \\
2
\end{array}\right)}\right)^{1+k-j} z^{n}
$$

The Askey-Wilson polynomials $p_{n}=p_{n}(x ; a, b, c, d ; q)$ are defined as follows:

$$
P_{n}(x ; a, b, c, d ; q)=\frac{(a b, a c, a d)_{n}}{a^{n}}{ }_{4} \phi_{3}\left[\begin{array}{c}
q^{-n}, a b c d q^{n-1}, a e^{i \theta}, a e^{-i \theta} \\
a b, a c, a d
\end{array} ; q, q\right]
$$$$
x=\cos \theta .
$$

The orthogonality relation for Askey-Wilson polynomials is

$$
\frac{1}{2 \pi} \int_{0}^{\pi} P_{n}(\cos \theta, a, b, c, d ; q) P_{m}(\cos \theta, a, b, c, d ; q) w(\cos \theta, a, b, c, d ; q) d \theta=0,
$$$$
n \neq m
$$

where

$$
w(\cos \theta, a, b, c, d ; q)=\frac{\left(e^{2 i \theta}, e^{-2 i \theta}\right)_{\infty}}{\left(a e^{i \theta}, a e^{-i \theta}, b e^{i \theta}, b e^{-i \theta}, c e^{i \theta}, c e^{-i \theta}, d e^{i \theta}, d e^{-i \theta}\right)_{\infty}} .
$$

We now introduce Motzkin paths, which are the objects used in the first combinatorial approach described below. It is a tool that we will use a lot throughout this paper. We are given a set of steps $S=\left\{s_{1}, s_{2}, \ldots, s_{k}\right\}$, such that each step $s_{i}$ has a direction, that can be up, down or horizontal, and a weight $\omega\left(s_{j}\right) \in \mathbb{R}$. In our context, the weight function may implicitly depend on fixed real parameters and on the height $i$ of the beginning of the step. A step going up applies a change to the height of 1, a step going down applies a change to the height of -1 , and a horizontal step applies a change to the height of 0 .

Now, a Motzkin Path of length $n$ on the set of steps $S$ is a sequence of $n$ steps in $S$ that starts at height 0 and ends at height 0 , and always keeping a nonnegative level. We will denote the set of such paths $\operatorname{Mot}_{n}(S)$, or $\operatorname{Mot}_{n}$ when there is no ambiguity on the set of steps. We extend the weight function $\omega$ to Motzkin paths by setting

$$
\omega(P)=\prod_{k=1}^{n} \omega\left(s_{j_{k}}\right), \text { for } P=\left(s_{j_{1}}, \ldots, s_{j_{n}}\right) .
$$

Throughout this paper, we will graphically represent the steps and the Motzkin paths as in figure 1. Note that the weight depends on the level and on the variables $a$ and $q$. 

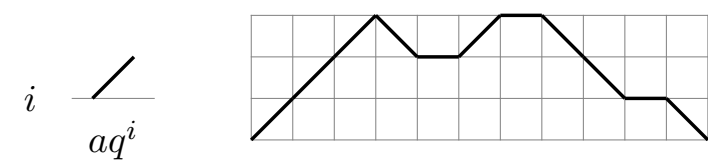

Figure 1: A step going up of weight $a q^{i}$ (left), a Motzkin path of size 11 (right).

\subsection{Three combinatorial approaches}

In this subsection, we present three combinatorial approaches that are studied for computing the $n$th moment $\mu=\mu_{n}(a, b, c, d ; q)$ of the Askey-Wilson polynomials. We only introduced the definitions and notations of the Motzkin paths approach, because this is the one that we will use in this paper. The other two approaches involve tools and techniques that we will not use and are for mainly information purposes. The results that they brought will allow us to see how our result compares, and complements the state of the art on the subject. In particular, we recall theorem 2 that gives the value of the moments in the most general case. Also, we state theorem 1 which gives a combinatorial proof for the moments, but in a less general case. This means that our result is the natural extension of theorem 1.

\subsubsection{First combinatorial approach: Motzkin paths}

It is known by Favard that any monic orthogonal polynomial $P_{n}(x)$ satisfies a three-term recurrence $P_{n+1}(x)=\left(x-b_{n}\right) P_{n}(x)-\lambda_{n} P_{n-1}(x)$.

Viennot [14] showed that the moment $\mu_{n}$ is the sum of weights of weighted Motzkin paths of length $n$ whose weights are defined using $b_{n}$ and $\lambda_{n}$. Viennot's theory provides a connection between orthogonal polynomials and combinatorics.

The monic Askey-Wilson polynomials $P_{n}=P_{n}(x ; a, b, c, d ; q)$ are defined by the threeterm recurrence $P_{n+1}=\left(x-b_{n}\right) P_{n}-\lambda_{n} P_{n-1}$ with $P_{-1}=0$ and $P_{0}=1$ for $b_{n}=\frac{1}{2}(a+$ $\left.a^{-1}-\left(A_{n}+C_{n}\right)\right)$ and $\lambda_{n}=\frac{1}{4} A_{n-1} C_{n}$, where

$$
\begin{aligned}
& A_{n}=\frac{\left(1-a b q^{n}\right)\left(1-a c q^{n}\right)\left(1-a d q^{n}\right)\left(1-a b c d q^{n-1}\right)}{a\left(1-a b c d q^{2 n-1}\right)\left(1-a b c d q^{2 n}\right)} \\
& C_{n}=\frac{a\left(1-q^{n}\right)\left(1-b c q^{n-1}\right)\left(1-b d q^{n-1}\right)\left(1-c d q^{n-1}\right)}{\left(1-a b c d q^{2 n-2}\right)\left(1-a b c d q^{2 n-1}\right)} .
\end{aligned}
$$

Since $b_{n}$ and $\lambda_{n}$ are complicated, it is difficult to compute $\mu_{n}$ using Motzkin paths. However, when $c=d=0$, we have $b_{n}=\frac{1}{2}(a+b) q^{n}$ and $\lambda_{n}=\left(1-q^{n}\right)\left(1-a b q^{n-1}\right)$. Kim and Stanton [10] found the following formula for $\mu_{n}(a, b, 0,0 ; q)$ using Motzkin paths:

\section{Theorem 1.}

$$
2^{n} \mu_{n}(a, b, 0,0 ; q)=\sum_{k=0}^{n}\left(\left(\begin{array}{c}
n \\
\frac{n-k}{2}
\end{array}\right)-\left(\begin{array}{c}
n \\
\frac{n-k}{2}-1
\end{array}\right)\right) \cdot \sum_{u+v+2 t=k} a^{u} b^{v}(-1)^{t} q^{\left(\begin{array}{c}
t+1 \\
2
\end{array}\right)}\left[\begin{array}{c}
u+v+t \\
u, v, t
\end{array}\right]_{q} .
$$




\subsubsection{Second combinatorial approach: staircase tableaux}

The staircase tableaux were first introduced by Corteel and Williams [4] and further studied in [3]. Corteel and Williams showed that the moment $\mu_{n}(a, b, c, d ; q)$ is a generating function for staircase tableaux of length $n$. They also showed that staircase tableaux have a connection with an important statistical mechanics model called asymmetric exclusion process (ASEP).

The weight of a staircase tableau, which is rather complicated, involves some complex numbers. Hence, it is not so easy to study $\mu_{n}$ using staircase tableaux. Using staircase tableaux, Kim and Stanton [10] showed that the coefficient of the highest term $a^{n} b^{n} c^{n} d^{n} q^{\left(\begin{array}{c}n \\ 2\end{array}\right)}$ in $2^{n}(a b c d)_{n} \mu_{n}(a, b, c, d ; q)$, which turns out to be a polynomial, is the Catalan number $\operatorname{Cat}(n / 2)$ if $n$ is even, and 0 otherwise.

It is worthwhile noting that staircase tableaux contain permutation

tableaux as special cases. Permutation tableaux are in bijection with permutations and there are many interesting bijections transforming certain statistics. Permutation tableaux have connections with total positivity in Grassmannian and a statistical mechanics model called partially asymmetric exclusion process (PASEP).

\subsubsection{Third combinatorial approach: $q$-Hermite polynomials}

The Askey-Wilson integral is

$$
I:=\frac{(q)_{\infty}}{2 \pi} \int_{0}^{\pi} w(\cos \theta, a, b, c, d ; q) d \theta=\frac{(a b c d)_{\infty}}{(a b, a c, a d, b c, b d, c d)_{\infty}}
$$

Ismail, Stanton, and Viennot [7] showed that the integral $I$ is a generating function for inhomogeneous matchings on four blocks $\left[n_{1}\right] \uplus\left[n_{2}\right] \uplus\left[n_{3}\right] \uplus\left[n_{4}\right]$ for $n_{1}, n_{2}, n_{3}, n_{4} \geqslant 0$ using $q$-Hermite polynomials. Here, "inhomogeneous" means that every edge connects two elements in different blocks. By enumerating this generating function they proved (2) combinatorially.

Kim and Stanton [11] recently found that their idea can be extended to compute the moment $\mu_{n}(a, b, c, d ; q)$ as follows. Let

$$
I_{n}:=\frac{(q)_{\infty}}{2 \pi} \int_{0}^{\pi}(\cos \theta)^{n} w(\cos \theta, a, b, c, d ; q) d \theta
$$

Then $I_{0}=I$ and $\mu_{n}(a, b, c, d ; q)=I_{n} / I$. In [11] they showed that $I_{n}$ is a generating function for matchings on five blocks $[n] \uplus\left[n_{1}\right] \uplus\left[n_{2}\right] \uplus\left[n_{3}\right] \uplus\left[n_{4}\right]$ for $n_{1}, n_{2}, n_{3}, n_{4} \geqslant 0$ in which homogeneous edges are only in the first block $[n]$. Using this combinatorial model, [11] found the following formula for the moment $\mu_{n}$.

Theorem 2 ([11]). We have

$$
2^{n} \mu_{n}(a, b, c, d ; q)=\sum_{k=0}^{n}\left(\left(\begin{array}{c}
n \\
\frac{n-k}{2}
\end{array}\right)-\left(\begin{array}{c}
n \\
\frac{n-k}{2}-1
\end{array}\right)\right) \sum_{\alpha+\beta+\gamma+\delta+2 t=k} a^{\alpha} b^{\beta} c^{\gamma} d^{\delta} \frac{(a c)_{\beta}(b d)_{\gamma}}{(a b c d)_{\beta+\gamma}}
$$




$$
\times(-1)^{t} q^{\left(\begin{array}{c}
++1 \\
2
\end{array}\right)}\left[\begin{array}{c}
\alpha+\beta+\gamma+t \\
\alpha
\end{array}\right]_{q}\left[\begin{array}{c}
\beta+\gamma+\delta+t \\
\beta, \gamma, \delta+t
\end{array}\right]_{q}\left[\begin{array}{c}
\delta+\alpha+t \\
\delta
\end{array}\right]_{q}
$$

If $c=0$ in the above theorem, we get the following theorem immediately after exchanging $c$ and $d$.

Theorem 3 ([10], [11]). The Askey-Wilson moments for $d=0$ are

$$
\begin{aligned}
2^{n} \mu_{n}(a, b, c, 0 ; q)=\sum_{k=0}^{n}\left(\left(\begin{array}{c}
n \\
\frac{n-k}{2}
\end{array}\right)-\left(\begin{array}{c}
n \\
\frac{n-k}{2}-1
\end{array}\right)\right) & \\
\cdot & \sum_{u+v+w+2 t=k} a^{u} b^{v} c^{w}(-1)^{t} q^{\left(\begin{array}{c}
(+1 \\
2
\end{array}\right)}\left[\begin{array}{c}
u+v+t \\
v
\end{array}\right]_{q}\left[\begin{array}{c}
v+w+t \\
w
\end{array}\right]_{q}\left[\begin{array}{c}
w+u+t \\
u
\end{array}\right]_{q} .
\end{aligned}
$$

Note that the proof of Theorem 3 is not completely combinatorial.

When $a c=b d=q$, then the moment becomes surprisingly simple.

Corollary 4 ([11]). We have

$$
\begin{aligned}
& 2^{n} \mu_{n}(a, b, q / a, q / b ; q)= \sum_{k=0}^{n}\left(\left(\begin{array}{c}
n \\
\frac{n-k}{2}
\end{array}\right)-\left(\begin{array}{c}
n \\
\frac{n-k}{2}-1
\end{array}\right)\right) \\
& \cdot\left(\frac{1}{[k+1]_{q}} \sum_{\substack{|A|+|B| \leqslant k \\
A+B \equiv k \bmod 2}} a^{A} b^{B} q^{\frac{k-A-B}{2}}\right),
\end{aligned}
$$

where the second sum is over all integers $A$ and $B$ such that $|A|+|B| \leqslant k$ and $A+B \equiv k$ $\bmod 2$.

This proves the following positivity conjectured in [10].

Their proof of Corollary 4 makes use of basic hypergeometric series.

\subsection{Goal of this article}

The theorem 1 which is a specific case of the theorem 3 has a combinatorial proof using Motzkin paths and Doubly Striped skew shapes. We recall the method as it will be close to ours, because it also uses a sign reversing involution argument. Since our goal is to find a combinatorial proof of theorem 3, we later briefly explain our strategy. This strategy will, in the next section, be explained further on the Al-Salam-Carlitz case.

\subsubsection{Presentation of the sign reversing involution method: the proof of the- orem 3}

Let us sketch the proof of theorem 1 done in [10] in order to show the idea that we will generalize. 
First, the connection provided by [14] leads to equation 3.

$$
\nu_{n}(a, b ; q)=\sum_{P \in \operatorname{Mot}(S)} \omega(P) .
$$

In the case $c=d=0, A_{n}=\frac{1-a b q^{n}}{a}$ and $C_{n}=a\left(1-q^{n}\right)$ give for $\lambda_{n}$ and $b_{n}$, the respective values $4 \lambda_{n}=\left(1-a b q^{n-1}\right)\left(1-q^{n}\right)$ and $2 b_{n}=(a+b) q^{n}$. Thereby leading to the possible steps $S$ described as in the figure 2 .

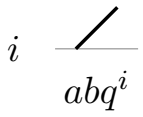

$i-\frac{}{b q^{i}}$

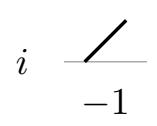

$i$

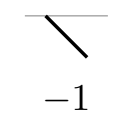

$i \overline{a q^{i}}$

$i$

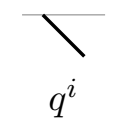

Figure 2: Available weighted steps

One can state the following thanks to a variation of Penaud's decomposition [12]:

$$
\sum_{P \in \operatorname{Mot}_{n}(a, b)} \omega(P)=\sum_{k=0}^{n}\left(\left(\begin{array}{c}
n \\
\frac{n-k}{2}
\end{array}\right)-\left(\begin{array}{c}
n \\
\frac{n-k}{2}-1
\end{array}\right)\right) \sum_{P \in \operatorname{Mot}_{k}^{*}(a, b)} \omega(P)
$$

Where $\operatorname{Mot}_{n}(a, b)$ denotes the Motzkin paths of size $n$ constituted of the steps shown in figure 2, and $\operatorname{Mot}_{n}^{*}(a, b)$ denotes the set of weighted Motzkin paths in $\operatorname{Mot}_{n}(a, b)$ such that there is no peak of weight 1 , that is an up step of weight -1 immediately followed by a down step of weight -1 .

In [10], Kim and Stanton then use a bijection $\psi$ between Motzkin paths and striped skew shapes. Striped skew shapes are objects that share similarities with Motzkin paths, we also similarly define a weight function for them. This bijection is explicitely defined in the section 3 of their paper. We give an example of the corresponding striped skew shape to a Motzkin path by the function $\psi$ in figure 3 .

Because $\psi$ preserves the weight, we obtain equation 4 .

$$
\sum_{P \in \operatorname{Mot}} \omega(P)=\sum_{S \in \mathrm{SSS}} \omega(S)
$$

where SSS stands for the set of Doubly Striped Skew Shapes.

In order to use a sign reversing involution argument, they define an involution $\phi$ acting on striped skew shapes. This function is a reversing sign involution, meanging that it satisfies the condition

$$
\left\{\begin{array}{l}
\omega(\phi(S))= \pm \omega(S) \\
\phi^{2}(S)=S
\end{array}\right.
$$


By a standard argument, the sum then simplifies to a sum over the set of fixpoints of $\phi$, as written in formula 5.

$$
\sum_{P \in \operatorname{Mot}_{k}^{*}(a, b)} \omega(P)=\sum_{S \in \text { Fixpoint }(\phi)} \omega(P) .
$$
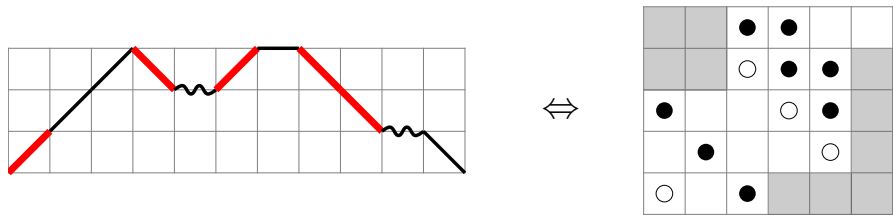

Figure 3: An example of the bijection $\psi$. The up steps of weight -1 and the down steps of weight -1 are colored red.
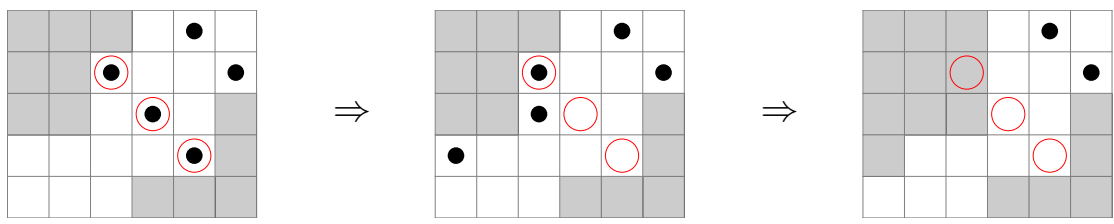

Figure 4: Example of the involution $\phi$.

Intuitively, the function $\phi$ will cancel every Motzkin paths that contains black dots or grey cases in the upper part.

The fixpoints of $\phi$ are the elements described in the figure 5, which are the striped skew shapes that only contains grey cases on the lower side, and white dots.

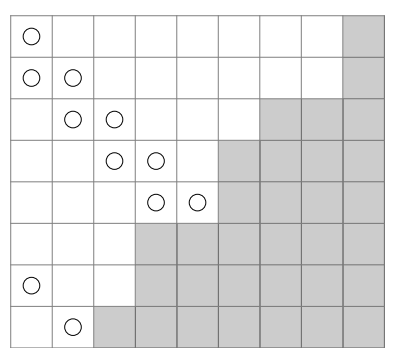

Figure 5: Fixpoint of $\phi$

At this stage, one needs to compute the weighted sum over the set of fixpoints. To compute the sum over the elements of fixpoint $(\phi)$ of the weighted striped skew shapes, a bijection $\delta$ between elements of fixpoint $(\phi)$ and words over $0,1,2$ is used. This bijection preserves weight if we consider that for $w=w_{1} \ldots w_{k^{\prime}}$, with $\mathrm{u} 0, \mathrm{v} 2$ and $\mathrm{t} 1$, weight $(w)=$ 


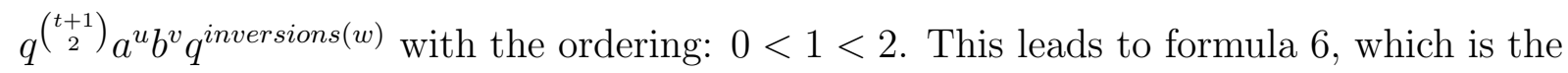
expected result.

$$
\sum_{i=0}^{k} \sum_{S \in D S S(i, k-i)} \omega_{a, b}(S)=\sum_{u+v+2 t=k} a^{u} b^{v}(-1)^{t} q^{\left(\begin{array}{c}
t+1 \\
2
\end{array}\right)}\left(\begin{array}{c}
u+v+t \\
u, v, t
\end{array}\right)_{q} .
$$

Remark 5. The function $\phi$ takes the black dot or the grey case on the left of minimal height, and then applies the change.(refer to [10] for a complete definition). The description of its action on Motzkin paths is very complicated and involves a lot of different cases (some possibilities on Doubly Striped Skew Shapes are shown in the figure 21 of the appendix).

\subsubsection{Method}

In term of Motzkin paths, we have

$$
\sum_{M \in \operatorname{Mot}_{k}^{*}(a, b)} \omega(P)=\sum_{M \in \psi^{-1}(\text { Fixpoint }(\phi))} \omega(M)
$$

Indeed, by calling $\tilde{\phi}=\psi^{-1} \circ \phi \circ \psi, \tilde{\phi}$ is also a sign reversing involution of fix points $\psi^{-1}($ Fixpoint $(\phi))$, a set that will now be referred to as $F(a, b)$.

A Motzkin path is a fixpoint of $\tilde{\phi}$ iff its image by $\psi$ is of the previously described form. The set of fixpoints of $\tilde{\phi}$ can also be described as follows. With the set of steps $A=\left\{a b q^{i}(\nearrow), a q^{i}(\rightarrow),-1(\nearrow)\right\}$ and $B=\left\{b q^{i}(\rightarrow), q^{i}(\searrow)\right\}$; a Motzkin path is in $F(a, b)$ if and only if it consists of elements in $A$, followed by only elements in $B$. Formally, for every $0 \leqslant i \leqslant u+t, s_{i} \in A$; and for every $u+t<i \leqslant u+v+2 t, s_{i} \in B$. Such paths are described in figure 6 .

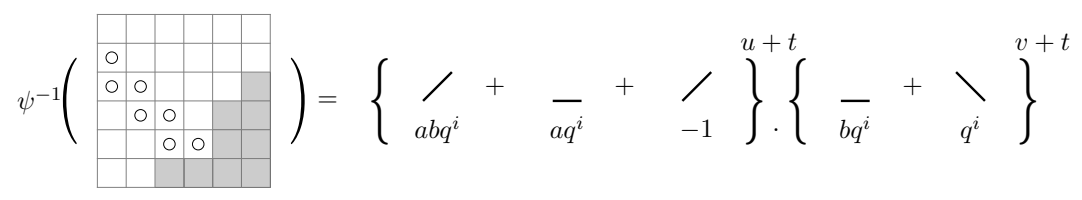

Figure 6: Fixpoints of $\tilde{\phi}$ with the notation used in [10]

The idea is to understand the bijection $\tilde{\phi}$ that can be defined in a more general case than $\phi$ as it is not restricted by the doubly striped skew shapes. To do so, we look at the direct effect of $\phi$, at the Motzkin path level instead of the striped skew shapes level.

We first introduced and solved another problem, to better our understanding of the problem and to introduce the paired words. Paired words will be structurally close to the set of fixpoints.

In [9], a similar combinatorial proof has been made for the Al-Salam-Carlitz moments (introduced in the next section) using simply striped skew shapes. In Section 2, we first give a combinatorial proof of Al-Salam-Carlitz moments using only Motzkin paths.

Later, we generalize this approach in section 3 to Askey-Wilson polynomials and make a proof of theorem 3, that is fully combinatorial. 


\section{Al-Salam Carlitz moments}

The Al-Salam Carlitz polynomials $U_{n}^{(a)}(x)$ (see [1]) are a family of orthogonal polynomials defined by the following generating function :

$$
\frac{(w)_{\infty}(a w)_{\infty}}{(x w)_{\infty}}=\sum_{n=0}^{\infty} U_{n}^{(a)}(x) \frac{w^{n}}{(q)_{n}}
$$

In this section, we prove the following using Motzkin path only, a result which was previously proven in [9] with striped skew shapes. (This proof is very similar to a more complicated one, done for Motzkin path moments where $c \neq 0$ in section 3 ).

If we denote by $L_{n}$ the $n^{\text {th }}$ moment of the Al-Salam-Carlitz polynomials, the result states that

$$
L\left(x^{n}\right)=\sum_{k=0}^{n}\left(\begin{array}{l}
n \\
k
\end{array}\right)_{q} a^{k} .
$$

\subsection{Definitions and intuition}

These polynomials satisfy the three terms recurrence relation:

$$
U_{n+1}^{(a)}(x)=\left(x-(1+a) q^{n}\right) U_{n}^{(a)}(x)+a q^{n-1}\left(1-q^{n}\right) U_{n-1}^{(a)}(x)
$$

Let us define $\operatorname{Mot}_{n}$, set of Motzkin paths over the following weighted steps:

$$
\begin{array}{ccccc}
a q^{i} & a q^{i} & q^{i} & q^{i} & -1 \\
\nearrow & - & - & \searrow & \searrow
\end{array}
$$

Figure 7: Available weighted steps

According to Viennot's theory [14], the Motzkin paths defined with the previously defined steps (figure 7) allow us to compute the $n^{\text {th }}$ moment, as described by formula 7 .

$$
\sum_{P \in \operatorname{Mot}_{n}} \omega(P)=L_{n}
$$

Definition 6. Let $A=\left\{a q^{i}(\uparrow), q^{i}(\rightarrow)\right\}, B=\left\{q^{i}(\downarrow), a q^{i}(\rightarrow)\right\}$ and $C=\{-1(\downarrow)\}$, as shown in figure 8.

Remark 7. The main idea in the following is to cancel all paths with a step of $B$ followed by a step of $A$ by switching the positions or finding an element of $A$ followed by an element of $B$ with the same weight, the gain of q exponent will be compensated by replacing a $q^{i}$ decreasing step by a -1 decreasing step. In order to remove all paths of $E_{1}$ and $E_{2}$ (defined later here 2.3), we need to define $\phi$ the following way. But let us first introduce the paired words formalism. 


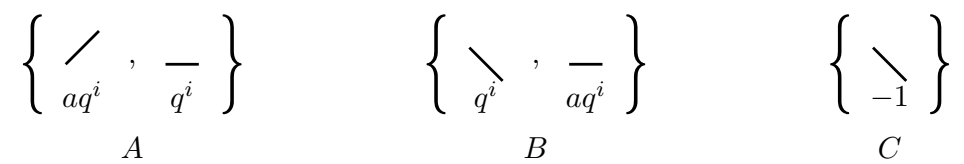

Figure 8: Definition of A, B and C

\subsection{Bijection with paired words}

In this subsection we introduce a formalism which is helpful to define a sign reversing involution and characterize its set of fixpoints.

One defines paired words on the alphabet $\{a, 1\}$ and the ordering $a<_{1} 1$ as a pair of:

- a word $w$ on the alphabet $\{a, 1\}$

- a set of pairs $F$ on the word $w$ which has to respect the following conditions

- $\forall(i, j) \in F, w_{i}<_{1} w_{j}$ (The pair has to respect the ordering between the letters)

- $(i, j) \in F \Rightarrow \nexists\left(i, j^{\prime}\right) \in F$ with $j \neq j^{\prime}$ and $(i, j) \in F \Rightarrow \nexists\left(i^{\prime}, j\right) \in F$ with $i \neq i^{\prime}$ (Each letter can be paired at most once)

The set of Motzkin paths without -1 down step is in bijection with paired words (bijection $\kappa$ briefly defined in figure 9); an example is given in figure 10 .

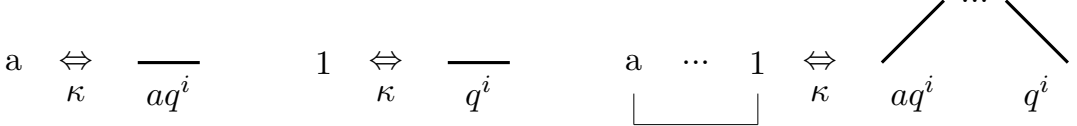

Figure 9: Equivalence between paired word and Motzkin paths

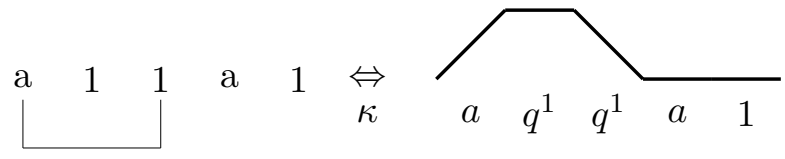

Figure 10: A path and its corresponding paired word

\subsection{Cancellation}

We define two functions $\phi_{1}: E_{1} \rightarrow E_{2}$ and $\phi_{2}: E_{2} \rightarrow E_{1}$ such that

- $\forall P \in E_{1}, w(P)=-w\left(\phi_{1}(P)\right)$

- $\forall P \in E_{2}, w(p)=-w\left(\phi_{2}(P)\right)$ 
- $\operatorname{Mot}_{n}=E_{1} \cup E_{2} \cup F$ where $\sum_{P \in F} w(P)$ is easy to compute.

Definition 8. Let $f$ be the function defined in figure $11 f: A \times(B \cup C) \rightarrow B \times A$.

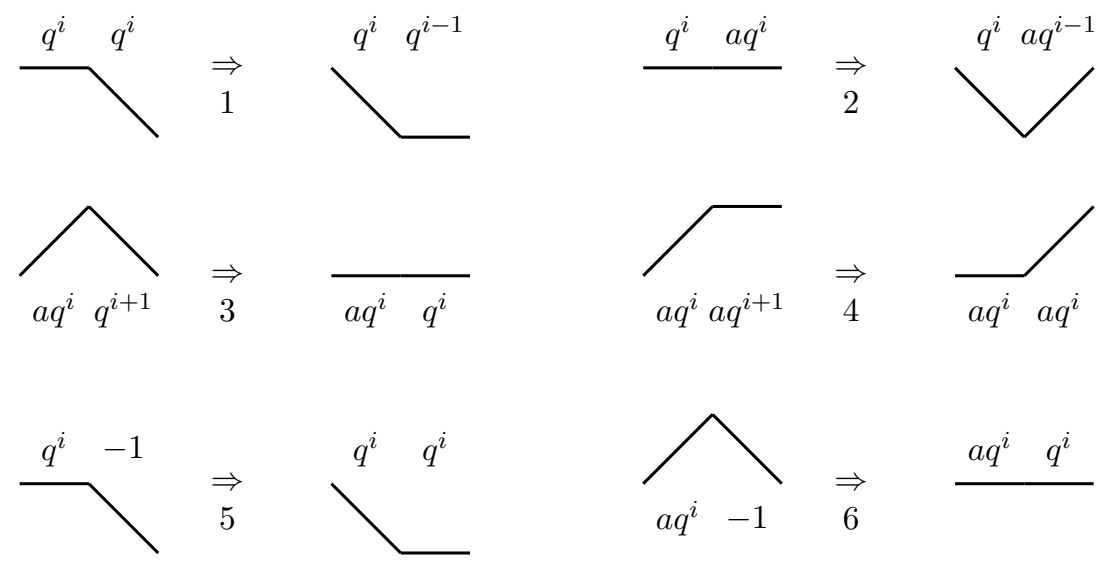

Figure 11: Definition of $f$

\section{Lemma 9.}

- For each $\left(w_{1}^{\prime}, w_{2}^{\prime}\right)=f\left(w_{1}, w_{2}\right)$ where $\left(w_{1}, w_{2}\right) \in A \times B, w\left(w_{1}\right) * w\left(w_{2}\right)=q * w\left(w_{1}^{\prime}\right) *$ $w\left(w_{2}^{\prime}\right)$

- For each $\left(w_{1}^{\prime}, w_{2}^{\prime}\right)=f\left(w_{1}, w_{2}\right)$ where $\left(w_{1}, w_{2}\right) \in A \times C, w\left(w_{1}\right) * w\left(w_{2}\right) * q^{i-1}=$ $w\left(w_{1}^{\prime}\right) * w\left(w_{2}^{\prime}\right)$

Definition of $\phi_{1}$ : Let $i_{0}=\min \left\{i \mid w_{i} \in C\right\}$. (the index of the -1 which will be replaced by a $q^{i}$ down step, thus $i$ iterations that multiplies by $q^{-1}$ must be done in order to compensate).

\section{- Initialisation:}

- $w_{i_{0}}$ is replaced by a $q^{i}$ step; weight is multiplied by $-q^{\text {level }\left(i_{0}\right)}$

- Induction: Let $i_{p+1}=\max \left\{i \mid i<i_{p} \& w_{i} \in A\right\}$. By induction, $w_{i_{p+1}} \in B$ so we can apply $f$ to $\left(w_{i_{p}}, w_{i_{p+1}}\right)$. The weight is multiplied by $q^{-1}$.

Lemma 10. We can do at least $i$ such described iterations.

Proof. Refer to E

This defines a function $\tilde{\phi}_{1}$ from $\operatorname{Mot}_{n}$ with a -1 step to $\operatorname{Mot}_{n}$. Let $j_{0}=\min \left\{j \mid w_{j} \in B\right\}$, and $j_{1}$ the index of the last modified step if $\psi_{1}$ was applied $(n+1$ if there is no step in $C)$.

Definition 11. $E_{1}=\left\{P \in M P \mid j_{1}<j_{0}\right\} E_{2}=\left\{P \in M P \mid j_{0}<j_{1}\right\}$ 
Since $\psi_{1}$ is trivially a bijection from $E_{1}$ to $E_{2}$, we can define $\psi_{2}=\psi_{1}^{-1}$ which is a bijection from $E_{2}$ to $E_{1}$. We then define $\psi: \operatorname{Mot}_{n} \rightarrow \operatorname{Mot}_{n}$

- $\psi(P)=\psi_{1}(P)$ if $P \in E_{1}$

- $\psi(P)=\psi_{2}(P)$ if $P \in E_{2}$

- $\psi(P)=P$ if $P \in F=M P \backslash\left(E_{1} \cup E_{2}\right)$

\section{Lemma 12.}

- $\forall P \in E_{1} \cup E_{2}, w(P)=-w(\psi(P))$

- $\psi \circ \psi=i d$

Before going further, one is advised to look at the full example given in the appendix $\mathrm{B}$ in order to fully understand how the function $\phi$ works.

\subsection{Maximal paired words and fixpoints}

In this subsection, we define an ordering between paired words which is total. The set of fixpoints for $\phi$ exactly corresponds to paired words such that their paired word is maximal for that ordering.

Definition 13. We define the ordering $\succ$ between set of pairs on a word as follows:

- If $F \subsetneq F^{\prime}$ then $F^{\prime} \succ F$

- If $F$ and $F^{\prime}$ are both maximal for the inclusion ordering then let $i_{1}$ be the smallest integer such that there exists $j_{0}$ such that $\left(i_{0}, j_{0}\right) \in F$ and $\left(i_{0}, j_{0}\right) \notin F^{\prime}$. Such an index always exists because otherwise the first case is applicable. If there exists no $j_{1}$ such that $\left(i_{0}, j_{1}\right) \in F^{\prime}$, then $F \succ F^{\prime}$ according to the first case. If there exists $j_{1}$ such that $\left(i_{0}, j_{1}\right) \in F^{\prime}$, then if $j_{0}=j_{1}$, it contradicts the definition of $i_{0}$. If $j_{0}<j_{1}$, $F^{\prime} \succ F$. If $j_{0}>j_{1}, F \succ F^{\prime}$.

Lemma 14. For each word, there exists only one pairing $T$ which is maximal for the previously defined ordering.

Proof. Let $T$ and $T^{\prime}$ be two maximal pairings over $w$.

- If $T$ or $T^{\prime}$ are not maximal for the inclusion ordering, we have a contradiction. Indeed this would mean that there exists no $(i, j)$ such that $(i, j)$ is a valid pair over $w$ and $i \notin\{i \mid(i, j) \in F\} \& j \notin\left\{j \mid(i, j) \in F^{\prime}\right\}$, thus $F \cup\{(i, j)\} \succ F$. Same for $F^{\prime}$.

- $\succ$ is clearly a total ordering among maximal sets due to the second item of the definition. Thus $T=T^{\prime}$.

With the previously stated result, we have $\sum_{P \in M P} w(P)=\sum_{P \in F} w(P)$, where $P \in$ $F \Leftrightarrow$ 
- $\nexists i, j$ such that $w_{i} \in B, w_{j} \in A, i<j$

- $\nexists i$ such that $w_{i} \in C$

Lemma 15. The function $\kappa$ is a bijection between $F$ and maximal paired words for $\succ$.

Remark 16. The proof that this is a bijection is not done here because a very similar and more complicated proof is already done for the case $c \neq 0$ in section 3 .

Remark 17. In order to obtain that set of fixpoints, $\phi$ takes a path with an element in $C$ and produces an element which is smaller than that path where -1 is replaced by $q^{i}$, thus it is not maximal (hence only path without step in $\mathrm{C}$ and maximal are fixpoints). This is the reason $f$ has been chosen that way.

Since the number of 1 is equal to the exponent of $a$, and the exponent of $q$ is equal to the number of inversion in the word (see section 3 for a more general proof), then

$$
\sum_{P \in F} w(P)=\sum_{\kappa(P), P \in F} a^{\# a(\kappa(P))} q^{i n v(\kappa(P))}=\sum_{k=0}^{n}\left(\begin{array}{l}
n \\
k
\end{array}\right)_{q} a^{k}
$$

\subsection{Theorem 3 is a generalization of the formula for Al-Salam-Carlitz mo- ments}

In this subsection, we see combinatorially why the theorem 3 is a generalization of the formula for Al-Salam-Carlitz moments.

As we will see in the next chapter, the Askey-Wilson moments are generated by the sum of the weighted Motzkin paths with the following possible steps.

The Al-Salam-Carlitz moments, are found by summing the weighted Motzkin paths with the following possible steps.

Since there is no up step with a weight of -1 available, this means that the procedure used to remove the peaks on weight 1 , and leading to the multiplicative factor $\sum_{k=0}^{n}\left(\left(\begin{array}{c}n \\ \frac{n-k}{2}\end{array}\right)-\left(\begin{array}{c}n \\ \frac{n-k}{2}-1\end{array}\right)\right)$, is not required anymore. Also, we can see that the set of available steps are a subset of the set of available steps for the Askey-Wilson polynomials, provided that $b=1$. The steps with a weight involving $c$ is not present. This means that moments are provided by the sum where the indexes $w$ and $t$ are always 0 .

When we chose $c=0$, the weight of the paths involving steps with a $c$ is zero. Hence, it is no surprise that without the multiplicative factor, the formula taking $b=1$ and $c=0$ coincides. Indeed, the formula provided by theorem 3 leads to

$$
\begin{aligned}
2^{n} \tilde{\mu}_{n}(a, 1,0,0 ; q)=\sum_{u+v+w=k} a^{u} b^{v} c^{w} q^{\left(\begin{array}{c}
t+1 \\
2
\end{array}\right)}\left[\begin{array}{c}
u+v \\
v
\end{array}\right]_{q}\left[\begin{array}{c}
v+w \\
w
\end{array}\right]_{q}\left[\begin{array}{c}
w+u \\
u
\end{array}\right]_{q} \\
=\sum_{u+v+w=k} a^{u} 1^{v} q^{\left(\begin{array}{c}
t+1 \\
2
\end{array}\right)}\left[\begin{array}{c}
u+v \\
v
\end{array}\right]_{q}\left[\begin{array}{l}
v \\
0
\end{array}\right]_{q}\left[\begin{array}{l}
u \\
u
\end{array}\right]_{q}=\sum_{u=0}^{k}\left(\begin{array}{l}
k \\
u
\end{array}\right) a_{q}^{u} .
\end{aligned}
$$




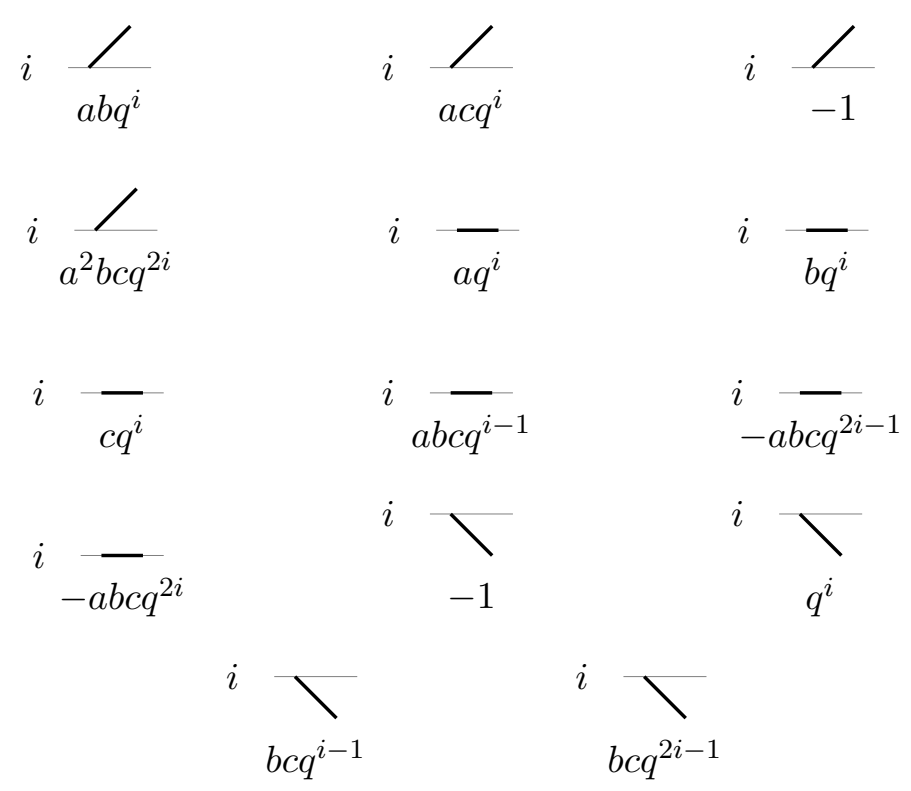

Figure 12: Available weighted steps for Askey-Wilson moments

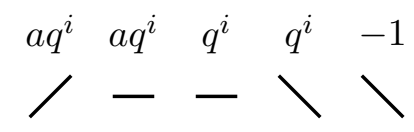

Figure 13: Available weighted steps for Al-Salam-Carlitz moments

\section{$3 \quad$ Askey-Wilson polynomials, case $c \neq 0$}

In this section, we give a fully combinatorial proof of the theorem 18 using Motzkin paths.

Theorem 18. ([10], [11]) The Askey-Wilson moments for $d=0$ are

$$
\begin{aligned}
2^{n} \mu_{n}(a, b, c, 0 ; q) & =\sum_{k=0}^{n}\left(\left(\begin{array}{c}
n \\
\frac{n-k}{2}
\end{array}\right)-\left(\begin{array}{c}
n \\
\frac{n-k}{2}-1
\end{array}\right)\right) \\
\cdot & \sum_{u+v+w+2 t=k} a^{u} b^{v} c^{w}(-1)^{t} q^{\left(\begin{array}{c}
t+1 \\
2
\end{array}\right)}\left[\begin{array}{c}
u+v+t \\
v
\end{array}\right]_{q}\left[\begin{array}{c}
v+w+t \\
w
\end{array}\right]_{q}\left[\begin{array}{c}
w+u+t \\
u
\end{array}\right]_{q}
\end{aligned}
$$

\subsection{Definitions}

The Askey-Wilson polynomials and the corresponding coefficients $A_{n}, C_{n}, \lambda_{n}$ and $b_{n}$ are defined as in the introduction 1.

The computation of the $A_{n}$ and $B_{n}$ coefficients (defined in [10]) gives the following. 


$$
\begin{gathered}
A_{n}=\frac{\left(1-a b q^{n}\right)\left(1-a c q^{n}\right)}{a} \text { and } C_{n}=a\left(1-q^{n}\right)\left(1-b c q^{n-1}\right), \text { which gives for } \lambda_{n} \text { and } b_{n} \\
4 \lambda_{n}=\left(1-a b q^{n-1}\right)\left(1-a c q^{n-1}\right)\left(1-b c q^{n-1}\right)\left(1-q^{n}\right) \\
=\left[-1+(a b+a c) q^{n-1}-a^{2} b c q^{2 n-1}\right]\left[-1+b c q^{n-1}-b c q^{2 n-1}\right]
\end{gathered}
$$

And

$$
b_{n}=(a+b+c) q^{n}+a b c q^{n}-a b c q^{2 n-1}(1+q)
$$

Hence, using Viennot's theory [14], we define Motzkin paths on the weighted steps described in figure 15 according to the value of $\lambda_{n}$ and $b_{n}$.

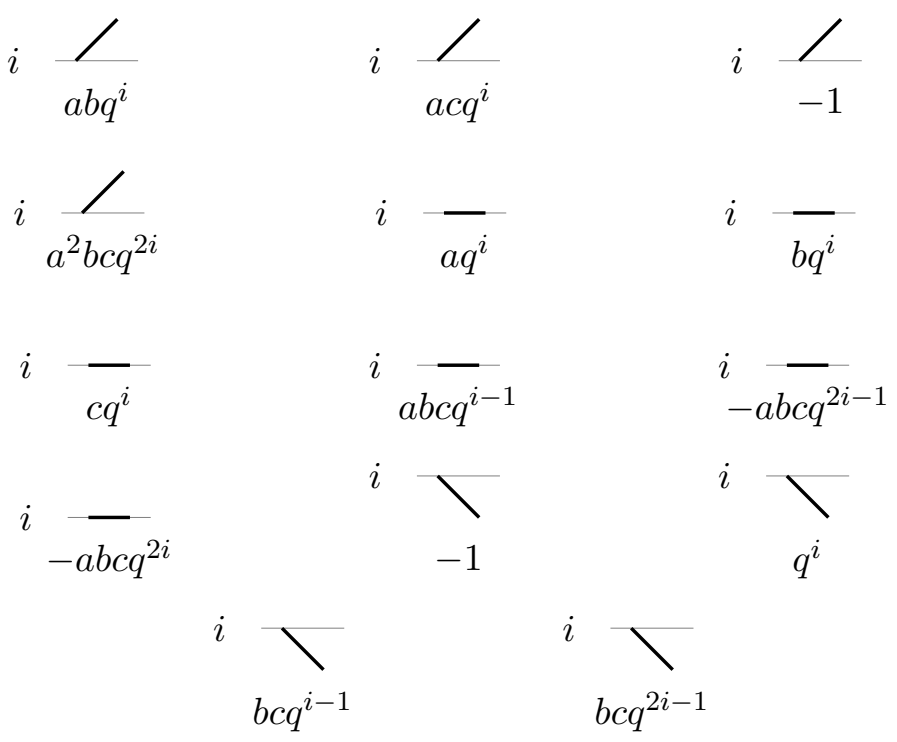

Figure 14: Available weighted steps

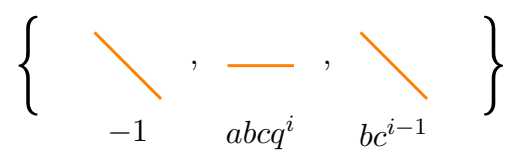

Figure 15: Definition of $C$

Remark 19. In the figure 15, we define the set of steps that will be used to remove most of the paths. They are the equivalent to the black dots steps in what has been done for $c=0$ in [10]. They will behave similarly as they have the same important property, namely $\forall s \in C, \exists s^{\prime}$ such that $w(s) *-q^{i}=w\left(s^{\prime}\right)$. So those steps will be used to cancel Motzkin path such that their weight can be increased by $q^{i}$. 
Definition 20.

$$
\begin{aligned}
& B S=\left\{a^{2} b c q^{2 i}(\uparrow),-a b c q^{i}(\rightarrow),-a b c q^{2 i}(\rightarrow),\right. \\
& \left.-a b c q^{2 i-1}(\rightarrow), b c q^{i}(\downarrow), b c q^{2 i-1}(\downarrow)\right\}
\end{aligned}
$$

- $B S^{\prime}=\left\{a^{2} b c q^{2 i}(\uparrow),-a b c q^{i}(\rightarrow),-a b c q^{2 i}(\rightarrow),-a b c q^{2 i-1}(\rightarrow), b c q^{2 i-1}(\downarrow)\right\}$

- $t_{2}=\# B S, t_{1}=\#\{-1(\uparrow),-1(\downarrow)\}$ and $t_{3}=\# B C^{\prime}\left(t_{1}, t_{2}\right.$ and $t_{3}$ implicitely depends of the considered path)

Definition 21. We denote by $\operatorname{Mot}_{n}$ the set of Motzkin paths of size $n$ built with steps in the figure 12 and by $\tilde{M o t}_{n}$ the set of paths that do not have a step in $C$ nor a $a b c q^{2 i}$ horizontal path.

Lemma 22. For $p$ Motzkin path, if $w(p)=a^{u} b^{v} c^{w} f(q)$, then $u+v+w+2\left(t_{1}-t_{2}\right)=n$. (Proven by counting the number of letters on each step).

\subsection{Bijection between words and Motzkin paths}

In this subsection, we create a link between words and Motzkin paths using paired words.

A paired word $w, F$ upon the alphabet $\{a, b, c, a b c,-1\}$ is a word $w$ and a set of couple of indexes $F$ such that,

$$
(i) \forall(i, j) \in F, w_{i}<w_{j}
$$

(for the ordering $a<b<c<a b c$ ), or

$$
w_{i}=w_{j}=a b c
$$

or

$$
w_{i}=w_{j}=-1
$$

Also,

$$
(i i) \forall i,(i, j) \in F \&\left(i, j^{\prime}\right) \in F \Rightarrow j=j^{\prime}
$$

If condition (i) is fulfilled, one will say that $F$ respects the ordering. If (ii) is fulfilled, all letters are paired at most once. Let us denote by $P W$ the set of all possible paired words on this alphabet (no length restriction).

We define the mapping $\kappa:$ Mot $_{n} \rightarrow P W$ as in the figure 16 .

Definition 23. We define the ordering $\succ$ between set of pairs on a word as follows:

- If $F \subsetneq F^{\prime}$ then $F^{\prime} \succ F$

- If $F$ and $F^{\prime}$ are both maximal for the inclusion ordering then let $i_{1}$ be the smallest integer such that there exists $j_{0}$ such that $\left(i_{0}, j_{0}\right) \in F$ and $\left(i_{0}, j_{0}\right) \notin F^{\prime}$. Such an index always exists because otherwise the first case is applicable. If there exists no $j_{1}$ such that $\left(i_{0}, j_{1}\right) \in F^{\prime}$, then $F \succ F^{\prime}$ according to the first case. If there exists $j_{1}$ such that $\left(i_{0}, j_{1}\right) \in F^{\prime}$, then if $j_{0}=j_{1}$, it contradicts the definition of $i_{0}$. If $j_{0}<j_{1}$, $F^{\prime} \succ F$. If $j_{0}>j_{1}, F \succ F^{\prime}$. 


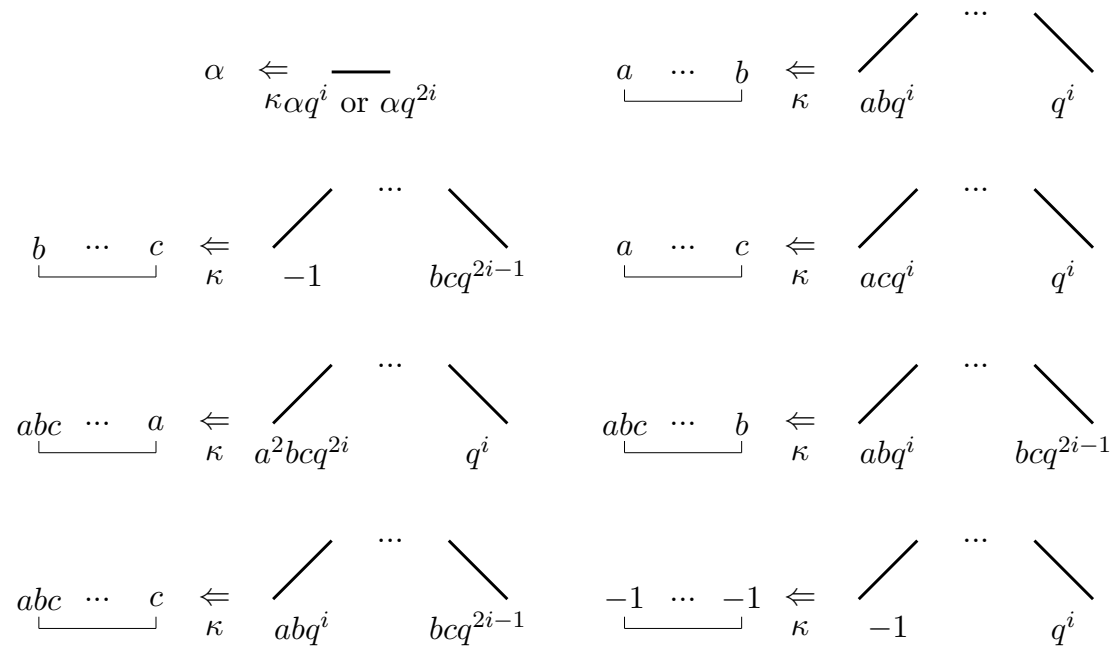

Figure 16: Correspondence between Motzkin paths and paired words

Lemma 24. $\succ$ is a total ordering.

Proof. See 2.4 .

Remark 25. There is no obvious relationships between the number of inversions in the paired word and the exponent of $q$ in the weight of the resulting Motzkin path, as we had in the case $c=0$. However, the Lemma 35 (defined and proved in the next section) allows us to deal with this issue.

$\kappa$ is a bijection between Motzkin paths without element in $C$ nor $a b c q^{2 i-1}$ horizontal step and paired words. However, it is not a bijection between Motzkin paths of size $n$ without element in $C$ nor $a b c q^{2 i-1}$ horizontal step and paired words of size $n$.

\section{Definition 26.}

- $P W(n)=\kappa \operatorname{Mot}_{n}$

- $W(n)=\{w \mid \exists(w, f) \in P W(n)\}$

\subsection{Involution and cancellation}

Let $f$ be the function defined in figure 17: $f: D_{f^{-1}} \rightarrow D_{f}$. In figure 17, $\alpha, \beta$ and $\gamma$ stand for the corresponding letter in the paired word that will be defined in section 3.4.

For a complete formal definition, please refer to appendix D.

Definition 27. $f^{-1}$ is clearly a bijection from $D_{f^{-1}}$ to $D_{f}$. Let $f$ be its reciprocal function. 


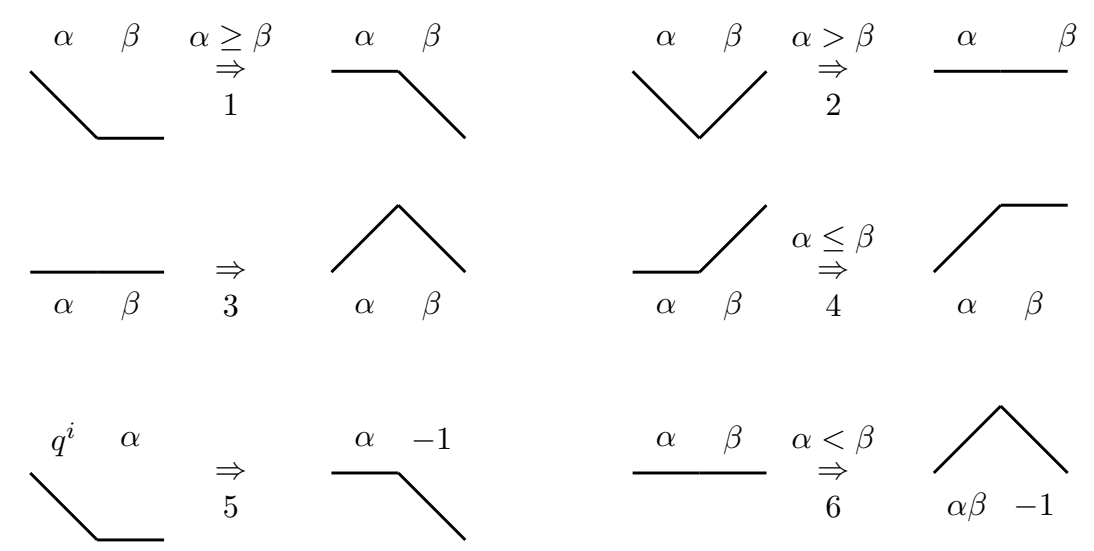

Figure 17: Informal definition of $f^{-1}$
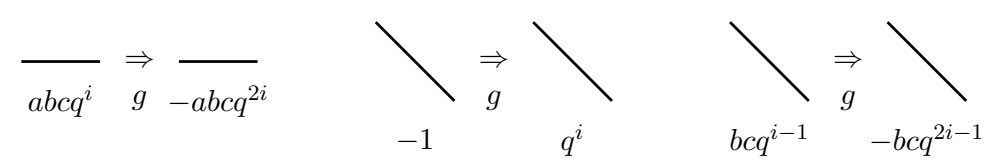

Figure 18: Definition of $g$

Remark 28. It is more intuitive to define $f^{-1}$ instead of defining $f$ directly; as it will be precisely defined in the 3rd item of the Lemma $29, f^{-1}$ 's action consists in taking a couple of steps for which the corresponding word is not maximal and produces a larger word for $\succ$.

We define $\phi$ as in the previous section. The only difference lies in function $f$, and $\nexists A, B$ such that $D_{f}=A \times B$. Intuitively, $f^{-1}$ is a function that takes two consecutive steps that do not correspond to a maximal pairing and corrects it. The image of the path after an iteration of $f^{-1}$ is always larger for $\succ$ (defined in section 3.2).

We define two functions $\phi_{1}: E_{1} \rightarrow E_{2}$ and $\phi_{2}: E_{2} \rightarrow E_{1}$ such that

- $\forall P \in E_{1}, w(P)=-w\left(\phi_{1}(P)\right)$

- $\forall P \in E_{2}, w(p)=-w\left(\phi_{2}(P)\right)$

- $M P=E_{1} \cup E_{2} \cup F$ where $\sum_{P \in F} w(P)$ is easy to compute.

\section{Lemma 29.}

- For each $\left(s_{1}^{\prime}, s_{2}^{\prime}\right)=f^{-1}\left(s_{1}, s_{2}\right)$ where $\left(s_{1}, s_{2}\right) \in D_{f}^{1}, s\left(s_{1}\right) * s\left(s_{2}\right)=q * s\left(s_{1}^{\prime}\right) * s\left(s_{2}^{\prime}\right)$.

- For each $w \in S, w(g(s))=-q^{i} w(s)$.

- (Key item) For each $P \in M P$, either $\kappa(P)$ is maximal for $\succ$, or $\exists(i, j)$ such that $\left(s_{i}, s_{j}\right) \in D_{f^{-1}}$ and, with $P^{\prime}$ the path such that $f^{-1}$ has been applied to $\left(s_{i}, s_{j}\right)$, 
$\kappa\left(\tilde{P}^{\prime}\right) \succ \kappa(\tilde{P})$. (Where $\left.\tilde{(} P\right)$ is the path where $g$ has been applied to each step if

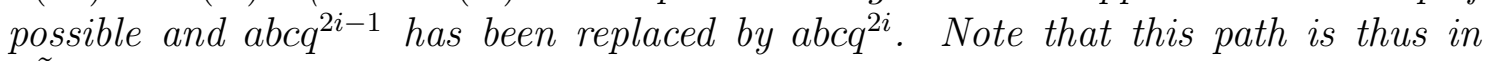
$\left.\tilde{M} P_{n}\right)$.

Proof. Item 1 and 2 directly come from the definition of $f$ and $g$. To prove the 3rd item, we use the complete definition of $f^{-1}$ given as the reverse of $f$ defined in appendix D. First, the definition of $f^{-1}$ is such that the corresponding resulting word w' (ie $\kappa\left(P^{\prime}\right)$ ) is equal to the initial word $\mathrm{w}($ ie $\kappa(P))$.

- If we can apply $f^{-1}$ :

- case (1), then it extends on the right a pair that could be extended.

- case (2), then it merges two different pairs to create a larger pair (which fulfills (ii)); the new pair is larger than the leftmost one so $(w, F)<\left(w, F^{\prime}\right)$.

- case (3), then it creates a new possible pair so $F \subset F^{\prime}$.

- case (4), then it extends on the left a pair that could be extended.

- Conversly, if $(w, F)$ is not maximal, let $\left(w, F^{\prime}\right)$ be a larger paired word:

- If $F \subset F^{\prime}$, then case (3) can be applied at least once (it does not necessarily give $F^{\prime}$, case (1) and (4) can be required afterward).

- If $\exists(i, j) \in F,\left(i, j^{\prime}\right) \in F^{\prime}$ and $j<j^{\prime}$, if $j+1$ is paired in $F$ then we can apply (2), else we can apply (1) to $(j, j+1)$.

- If $\exists(i, j) \in F,\left(i^{\prime}, j\right) \in F^{\prime}$ and $i^{\prime}<i$, if $i-1$ is paired in $F$ then we can apply $(2)$, else we can apply (4) to $(i-1, i)$.

Definition of $\phi_{1}$ : Let $i_{0}=\min \left\{i \mid w_{i} \in C\right\}$. (the index of the -1 which will be replaced by a $q^{i}$ down step, thus $i$ iterations that multiplies by $q^{-1}$ must be done in order to compensate).

- Initialisation: Apply $g$ to $s_{i_{0}}$; weight is multiplied by $-q^{\text {level }\left(i_{0}\right)}$.

- Induction: Let $i_{p+1}=\max \left\{i \mid i<i_{p} \&\left(w_{i}, w_{i+1} \in D_{f}\right.\right.$ or $\left.w_{i}=a b c q^{2 i}\right\}$.

- If $w_{i}=a b c q^{2 i}, w_{i}$ becomes $a b c q^{2 i-1}$; weight is multiplied by $q^{-1}$

- Else, apply $f$ to $\left(w_{i_{p+1}}, w_{i_{p+1}+1}\right)$; weight is multiplied by $q^{-1}$

This defines a function $\tilde{\phi}_{1}$ from $M P$ with a -1 step to $M P$. Let $j_{0}=\min \left\{j \mid w_{j} \in B\right\}$, and $j_{1}$ the index of the last modified step if $\psi_{1}$ was applied $(n+1$ if there is no step in $C)$.

Definition 30. $E_{1}=\left\{P \in M P \mid j_{1}<j_{0}\right\} E_{2}=\left\{P \in M P \mid j_{0}<j_{1}\right\}$ 
Since $\psi_{1}$ is obviously a bijection from $E_{1}$ to $E_{2}$, we can define $\psi_{2}=\psi_{1}^{-1}$ which is a bijection from $E_{2}$ to $E_{1}$. We then define $\psi: \operatorname{Mot}_{n} \rightarrow \operatorname{Mot}_{n}$

- $\psi(P)=\psi_{1}(P)$ if $P \in E_{1}$

- $\psi(P)=\psi_{2}(P)$ if $P \in E_{2}$

- $\psi(P)=P$ if $P \in F=M P \backslash\left(E_{1} \cup E_{2}\right)$

Lemma 31. • $\forall P \in E_{1} \cup E_{2}, w(P)=-w(\psi(P))$

- $\psi \circ \psi=i d$

- $F \subset \tilde{M P}$

- $F=\left\{P \in \tilde{M} P \mid \nexists(i, j),\left(s_{i}, s_{j}\right) \in D_{f^{-1}}\right\}$

Thanks to Lemma 29 and Lemma 31, the following result holds:

$$
\sum_{P \in \operatorname{Mot}_{n}} \omega(P)=\sum_{w \in W(n)} \omega(w)
$$

Where $\omega(w)$ is the weight of $\kappa(w, F)$ where $(w, F)$ is the only maximal paired word on $w$ (using Lemma 24).

Since $W(n)$ does not contain words of constant size, we can not compute this sum directly. In the next section, we make a bijection from triple of words with a specific size to $W(n)$ to solve this problem.

\subsection{Construction of $\gamma$}

Let us consider

$$
M_{u, v, w, t}(a, b, c) \quad=\quad\left[W_{a, b}(u, v+t) \times W_{b, c}(v, w+t) \times W_{c, a}(w, u+t)\right] .
$$

Lemma 32. $M_{u, v, w, t}(a, b, c)$ is a free monoid on the set $\{(a, \epsilon, a),(b, b, \epsilon),(\epsilon, c, c),(a, b, c),(b, c, a)\}$

Proof. To reconstruct the only decomposition, one can read the triple of word from left to right. If there is a majority (two times the same letter), then it is $(a, \epsilon, a),(b, b, \epsilon)$ or $(\epsilon, c, c)$. In the case there is no majority, each letter occurs exactly once which means it is $(a, b, c)$ or $(b, c, a)$.

The previous lemma allows us to define the following function. $\gamma: M_{u, v, w, t}(a, b, c) \rightarrow$ $W_{a, b, c, a b c,-1}$

- $\gamma((a, \epsilon, a))=a$

- $\gamma((b, b, \epsilon))=b$ 
- $\gamma((\epsilon, c, c))=c$

- $\gamma((a, b, c))=(a b c)$

- $\gamma((b, c, a))=(-1)$

And $\gamma$ is a morphism. (Thus entirely and uniquely defined).

Lemma 33. (i) For every word $w$ in $W_{a, b, c, a b c,-1}$, there exists a triple $w^{\prime}=\left(w_{1}, w_{2}, w_{3}\right)$ such that $\gamma\left(w^{\prime}\right)=w$. (ii) For every two triple of words $w_{1}^{\prime}$ and $w_{2}^{\prime}, w_{1}^{\prime} \neq w_{2}^{\prime} \Rightarrow \gamma\left(w_{1}^{\prime}\right) \neq$ $\gamma\left(w_{2}^{\prime}\right)$. Which means $\gamma$ is a bijection between all words and all triple of words.

Proof. Let $\tilde{\gamma}: M_{u, v, w, t} \rightarrow W_{a, b, c, a b c,-1}$ be the following function:

- $\tilde{\gamma}(a)=(a, \epsilon, a)$

- $\tilde{\gamma}(b)=(b, b, \epsilon)$

- $\tilde{\gamma}(c)=(\epsilon, c, c)$

- $\tilde{\gamma}(a b c)=(a, b, c)$

- $\tilde{\gamma}(-1)=(b, c, a)$

- And such that $\tilde{\gamma}\left(w_{1} \alpha\right)=\tilde{\gamma}\left(w_{1}\right) \tilde{\gamma}(\alpha)$ for $\alpha \in\{a, b, c, a b c,-1\}$.

By construction, and because of the free monoid property, $\gamma \circ \tilde{\gamma}(w)=w$ and $\tilde{\gamma} \circ \gamma(w)=w$, which proves (i) and (ii).

Lemma 34. With $\gamma$ the previously defined function between

$\{(a),(b),(c),(-a b c),(-1)\}$ and triple of words, if $\gamma(w)=\left(w_{1}, w_{2}, w_{3}\right)$, with $u=\#_{a}(w)$, $v=\#_{b}(w), w=\#_{c}(w), t_{1}=\#_{-1}(w), t_{2}=\#_{-a b c}(w)$ and $t=t_{1}-t_{2}$, then

$$
\begin{array}{ll}
\#_{a}\left(w_{1}\right)=u & \#_{b}\left(w_{1}\right)=v+t \\
\#_{b}\left(w_{2}\right)=v & \#_{c}\left(w_{2}\right)=w+t \\
\#_{c}\left(w_{1}\right)=w & \#_{a}\left(w_{3}\right)=u+t
\end{array}
$$

Proof. By induction on the word w. (In the following, $\epsilon$ stands for the empty word)

- If $w=\epsilon$, then $\gamma(w)=(\epsilon, \epsilon, \epsilon)$.

- If $w^{\prime}=w \cdot a$ then $\gamma\left(w^{\prime}\right)=\left(w_{1}, w_{2}, w_{3}\right) \cdot(a, \epsilon, a) u^{\prime}=u+1, v^{\prime}=v, w^{\prime}=w, t^{\prime}=t$.

$$
\begin{gathered}
\#_{a}\left(w_{1}^{\prime}\right)=\#_{a}\left(w_{1}\right)+1=u^{\prime} \quad \#_{b}\left(w_{1}^{\prime}\right)=v^{\prime}+t^{\prime} \\
\#_{b}\left(w_{2}^{\prime}\right)=\#_{b}\left(w_{2}\right)=v^{\prime} \quad \#_{c}\left(w_{2}^{\prime}\right)=w^{\prime}+t^{\prime} \\
\#_{c}\left(w_{1}^{\prime}\right)=\#_{c}\left(w_{3}\right)=w^{\prime} \quad \#_{a}\left(w_{3}^{\prime}\right)=\# a\left(w_{3}\right)+1=u^{\prime}+t^{\prime}
\end{gathered}
$$


Other cases are similar. For the full proof, please refer to appendix F

Lemma 35. With $n_{1}=\#\left\{(i, j) \mid w_{i}=b, w_{j}=a b c \& i<j\right\}$ and $n_{2}=\#\left\{(i, j) \mid w_{i}=b, w_{j}=\right.$ $a b c \& i>j\}$; for $P \in F_{a, b, c}$ such that $w(p)=a^{u} b^{v} c^{w} q^{m}$, and $\gamma(P)=\left(w_{1}, w_{2}, w_{3}\right)$ then $m=\left(\begin{array}{c}t_{1}-t_{2}+1 \\ 2\end{array}\right)+i n v_{1}\left(w_{1}\right)+i n v_{2}\left(w_{2}\right)+i n v_{3}\left(w_{3}\right)+n_{1}-n_{2}$. Where $i n v_{1}(w)=\#\{(i, j) \mid w(i)=$ $a \& w(j)=b\}, \operatorname{inv}_{2}(w)=\#\{(i, j) \mid w(i)=b \& w(j)=c\}, \operatorname{inv}_{3}(w)=\#\{(i, j) \mid w(i)=c \&$ $w(j)=a\}$. (One should be careful about the fact that the inversion definition is not the intuitive one and should refer to example 36).

The proof of Lemma 35 is done in Section 4.

Example 36. Let $W=(a a b, b c, c a a)$. Then

$$
\begin{aligned}
& \begin{array}{r}
u=2 \quad v=1 \quad w=1 \quad t=0 \quad n=4 \\
\gamma(W)=\gamma((a, b, c)) \cdot \gamma((a b, c, a a)) \\
=(a b c) \cdot \gamma((a, \epsilon, a)) \cdot \gamma((b, c, a)) \\
=(a b c) a(-1)
\end{array} \\
& \delta^{-1}((a b c) a(-1)=(-1)(a b c)(a)(-1)
\end{aligned}
$$

Then, the only maximal pairing is

$$
\{(1,4),(2,3)\}
$$

Which gives the following Motzkin path $\left(\right.$ in $\left.F_{a, b, c}\right)$ :

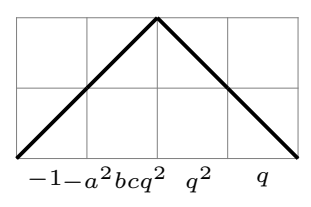

In total, the weight is

$$
a^{2} b c q^{2} * q^{2} * q=a^{2} b c q^{5}=a^{u} b^{v} c^{w} q^{i n v_{1}+i n v_{2}+i n v_{3}+n_{1}-n_{2}}
$$

Indeed,

$$
\begin{gathered}
i n v_{1}(a a b)=2 \quad \operatorname{inv}_{2}(b c)=1 \quad i n v_{3}(c a a)=2 \\
n_{1}=0 \quad n_{2}=0
\end{gathered}
$$




\subsection{Concluding the proof}

Equation 9 states that

$$
\sum_{P \in \operatorname{Mot}_{n}} \omega(P)=\sum_{w \in W(n)} \omega(w)
$$

Where $\omega(w)$ is the weight of $\kappa(w, F)$ where $(w, F)$ is the only maximal paired word on $w$ (using Lemma 24).

Also,

$$
\begin{aligned}
\sum_{w \in W(n)} \omega(w)= & \sum_{\left(w_{1}, w_{2}, w_{3}\right) \in \gamma(W(n))} \omega\left(\gamma^{-1}\left(w_{1}, w_{2}, w 3\right)\right) \\
& =\sum_{u+v+w+2 t=n} \sum_{\left(w_{1}, w_{2}, w_{3}\right) \in M_{u, v, w, t}}\left(\omega\left(\gamma^{-1}\left(w_{1}, w_{2}, w 3\right)\right)\right.
\end{aligned}
$$

Thanks to Lemma 34. Also, because of Lemma 35, if $\left(w_{1}, w_{2}, w_{3}\right) \in M_{u, v, w, t}$, then

$$
\omega\left(\gamma^{-1}\left(w_{1}, w_{2}, w 3\right)\right)=a^{u} b^{v} c^{w}(-1)^{t} q^{\left(\begin{array}{c}
++1 \\
2
\end{array}\right)} q^{i n v_{1}+i n v_{2}+i n v_{3}}
$$

Thus,

$$
\begin{aligned}
& \sum_{u+v+w+2 t=n} \sum_{\left(w_{1}, w_{2}, w_{3}\right) \in M_{u, v, w, t}}\left(\omega\left(\gamma^{-1}\left(w_{1}, w_{2}, w_{3}\right)\right)\right. \\
= & \sum_{u+v+w+2 t=n} a^{u} b^{v} c^{w}(-1)^{t} q^{\left(\begin{array}{c}
t+1 \\
2
\end{array}\right)} \sum_{w_{1} \in W_{a, b}(u, v+t)} q^{i n v_{1}\left(w_{1}\right)} \cdot \sum_{w_{2} \in W_{b, c}(v, w+t)} q^{i n v_{2}\left(w_{2}\right)} \sum_{w_{3} \in W_{c, a}(w, u+t)} q^{i n v_{3}\left(w_{3}\right)} \\
= & \sum_{u+v+w+2 t=n} a^{u} b^{v} c^{w}(-1)^{t} q^{\left(\begin{array}{c}
t+1 \\
2
\end{array}\right)} \cdot\left(\begin{array}{c}
u+v+t \\
u
\end{array}\right)_{q}\left(\begin{array}{c}
v+w+t \\
v
\end{array}\right)_{q}\left(\begin{array}{c}
u+w+t \\
w
\end{array}\right)_{q}
\end{aligned}
$$

Hence,

$$
\begin{aligned}
\sum_{P \in M P_{a, b, c}^{n}} w(P) & =\sum_{k=0}^{n}\left(\left(\begin{array}{c}
n \\
\frac{n-k}{2}
\end{array}\right)-\left(\begin{array}{c}
n \\
\frac{n-k}{2}-1
\end{array}\right)\right) \\
\cdot & \sum_{u+v+w+2 t=n} a^{u} b^{v} c^{w}(-1)^{t} q^{\left(\begin{array}{c}
t+1 \\
2
\end{array}\right)}\left(\begin{array}{c}
u+v+t \\
u
\end{array}\right)_{q}\left(\begin{array}{c}
v+w+t \\
v
\end{array}\right)_{q}\left(\begin{array}{c}
u+w+t \\
w
\end{array}\right)_{q}
\end{aligned}
$$

\section{Proof of Lemma 35 with crossings}

Recall:

$$
\begin{aligned}
& n_{1}=\#\left\{(i, j) \mid w_{i}=b, w_{j}=a b c \& i<j \& j \text { is not paired }\right\} \\
& n_{2}=\#\left\{(i, j) \mid w_{i}=b, w_{j}=a b c \& i>j \& i \text { is not paired }\right\}
\end{aligned}
$$

In this section, we prove Lemma 35 using crossings, a formalism on paired words which is introduced to simplify the proof. This proof could equivalently be done directly on Motzkin paths by considering the sum of the level of all steps rather than the crossings. 
Lemma 37. For $P \in F_{a, b, c}$ such that $w(p)=a^{u} b^{v} c^{w} q^{m}$, and $\gamma(P)=\left(w_{1}, w_{2}, w_{3}\right)$ then $m=\left(\begin{array}{c}t_{1}-t_{2}+1 \\ 2\end{array}\right)+i n v_{1}\left(w_{1}\right)+i n v_{2}\left(w_{2}\right)+i n v_{3}\left(w_{3}\right)+n_{1}-n_{2}$. Where inv $v_{1}(w)=$ $\#\{(i, j) \mid w(i)=a \& w(j)=b\}, i n v_{2}(w)=\#\{(i, j) \mid w(i)=b \& w(j)=c\}, i n v_{3}(w)=\#\{(i, j) \mid w(i)=c \&$ $w(j)=a\}$.

Remark 38. The corrective terms $n_{1}$ and $n_{2}$ simplify when we sum over all possible triple of words, because of a trivial involution that cancels them (for every pair with $\alpha=a b c$, reverse with the first $b$ inside the pair if it exists, for every pair with $\alpha=b$, reverse with the first $a b c$ inside the pair if it exists).
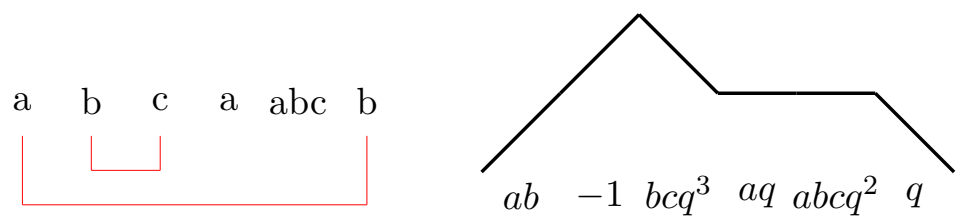

Figure 19: A maximal paired word and its corresponding Motzkin path

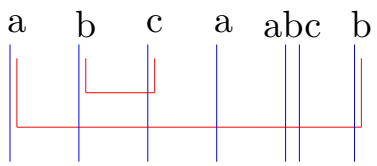

$\mathrm{Nb}$ of crossings $0+1+2+1+2+1=7$

Figure 20: The associated crossings

Definition 39. We graphically represent the paired words such as in the previous figure. For each letter $\gamma$ in the word $w$ :

- draw a vertical line if $\gamma \in\{a, b, c\}$

- draw two vertical lines if $\gamma \in\{a b c\}$

- draw a vertical line if $\gamma \in\{-1\}$ and is the right element of a pair. (Thus, each couple of paired -1 only have one vertical line)

This drawing leads to the previously shown figure. We call number of crossings the number of crossing of those vertical lines (in blue) and the pairing horizontal lines (in red).

Remark 40. As shown in the figure, the vertical line is "slightly on the left", which means that if $\gamma$ is a left element of a pair (ie $\exists \delta,(\gamma, \delta) \in F$ ) then the crossing with this pair is not counted. Also, if $\gamma$ is a right element of a pair (ie $\exists \delta,(\delta, \gamma) \in F$ ), the crossing with this pair is counted.

Remark 41. The number of crossings in the word after the number of -1 has been doubled, is also equal to the exponent of q in the corresponding Motzkin path (trivial). Hence, the previous lemma is equivalent to the following: For $w$ paired word, (number of crossings) $=\left(\begin{array}{c}t_{1}-t_{2}+1 \\ 2\end{array}\right)+($ number of inversions).

$$
\begin{array}{r}
\left(\begin{array}{c}
a \\
\epsilon \\
a
\end{array}\right) \Leftrightarrow a \quad\left(\begin{array}{c}
b \\
b \\
\epsilon
\end{array}\right) \Leftrightarrow b \quad\left(\begin{array}{c}
\epsilon \\
c \\
c
\end{array}\right) \Leftrightarrow c \\
\left(\begin{array}{c}
a \\
b \\
c
\end{array}\right) \Leftrightarrow a b c \quad\left(\begin{array}{c}
b \\
c \\
a
\end{array}\right) \Leftrightarrow(-1)
\end{array}
$$


Proof. First, let us make some remarks which will be useful for the proof of the lemma. For convenience, let us define $i n v=\left(i n v_{1}+i n v_{2}+i n v_{3}\right)+n_{1}-n_{2}$ (which is not the number of inversion in the resulting word).

- (i) if $\alpha<\beta$ for the order $a<b<c$, then inv $(\alpha \beta)=1$

- (ii) if $\alpha>\beta$ for the order $a<b<c$, then $\operatorname{inv}(\alpha \beta)=0$

- (iii) $\forall \alpha \in\{a, b, c, a b c,-1\}$, inv $(\alpha \alpha)=0$

- (iv) if $\alpha<\beta$ for the order $a<b<c$, then $\operatorname{inv}\left(\alpha\left(\begin{array}{c}a \\ b \\ c\end{array}\right) \beta\right)=3$

- (v) $\forall \alpha \in\{a, b\}, \operatorname{inv}\left(\alpha\left(\begin{array}{l}b \\ c \\ a\end{array}\right)\right)=1$

In the following, $\left(\begin{array}{c}w_{1} \\ w_{2} \\ w_{3}\end{array}\right)$ will stand for an element of

$$
\left[W_{a, b}(u, v+t) \times W_{b, c}(v, w+t) \times W_{c, a}(w, u+t)\right],
$$

$w$ the corresponding word in $W_{a, b, c, a b c,-1}\left(u-t_{2}, v-t_{2}, w-t_{2}, t_{2}, t_{1}\right)$ and $\delta(w)$ the corresponding word in $W_{a, b, c, a b c,-1}\left(u-t_{2}, v-t_{2}, w-t_{2}, t_{2}, 2 t_{1}\right)$. By induction on the pairs, with the hypothesis: $i n v_{1}\left(w_{1}\right)+$ $i n v_{2}\left(w_{2}\right)+i n v_{3}\left(w_{3}\right)+n_{1}-n_{2}+\left(\begin{array}{c}t_{1}-t_{2}+1 \\ 2\end{array}\right)=($ number of crossings $)(\delta(w))$.

- If $w$ does not contain any pair, according to the fact that the pairing should be maximal for the previously defined pairing rules, this means the number of inversion is also 0 , as the number of -1 . The number of $a b c$ is 0 or 1 , because if it is greater than 2, at least $2 a b c$ could be paired together.

- If $w^{\prime}=\alpha w \beta$, with $\alpha, \beta \in\{a, b, c\}$, where $w$ is a word with $m$ letters in $\{a, b, c\}, t_{1}$ in $\{-1\}$ and $t_{2}$ in $\{a b c\}$.

$$
\begin{aligned}
i n v^{\prime} & =i n v\left(\alpha\left(\begin{array}{c}
a \\
\epsilon \\
a
\end{array}\right)^{m}\left(\begin{array}{l}
b \\
c \\
a
\end{array}\right)^{t_{1}}\left(\begin{array}{l}
a \\
b \\
c
\end{array}\right)^{t_{2}} \beta\right)-i n v\left(\left(\begin{array}{l}
a \\
\epsilon \\
a
\end{array}\right)^{m}\left(\begin{array}{l}
b \\
c \\
a
\end{array}\right)^{t_{1}}\left(\begin{array}{l}
a \\
b \\
c
\end{array}\right)^{t_{2}}\right) \\
& =m+t_{1}+2 * t_{2}+1
\end{aligned}
$$

( $a$ and $b$ behaves the same according to (iv)). Also,

$$
\begin{aligned}
\text { crossings }^{\prime}=\text { crossings }\left(\alpha\left(\begin{array}{l}
a \\
\epsilon \\
a
\end{array}\right)^{m}\left(\begin{array}{l}
b \\
c \\
a
\end{array}\right)^{t_{1}}\left(\begin{array}{l}
a \\
b \\
c
\end{array}\right)^{t_{2}} \beta\right) & - \text { crossings }\left(\left(\begin{array}{l}
a \\
a \\
\epsilon
\end{array}\right)^{m}\left(\begin{array}{l}
b \\
c \\
a
\end{array}\right)^{t_{1}}\left(\begin{array}{l}
a \\
b \\
c
\end{array}\right)^{t_{2}}\right)
\end{aligned}
$$

Which is what we want, indeed:

- Other cases are similar but not exactly the same. We really recommand to read appendix G for the complete proof.

Remark 42. We have not considered the (-1) up steps that are created by $\delta$ except for the case $\alpha=-1$, $\beta=-1$ because the $q$ exponent is 0 so there is no line going down from them, thus it does not change the number of crossings. 


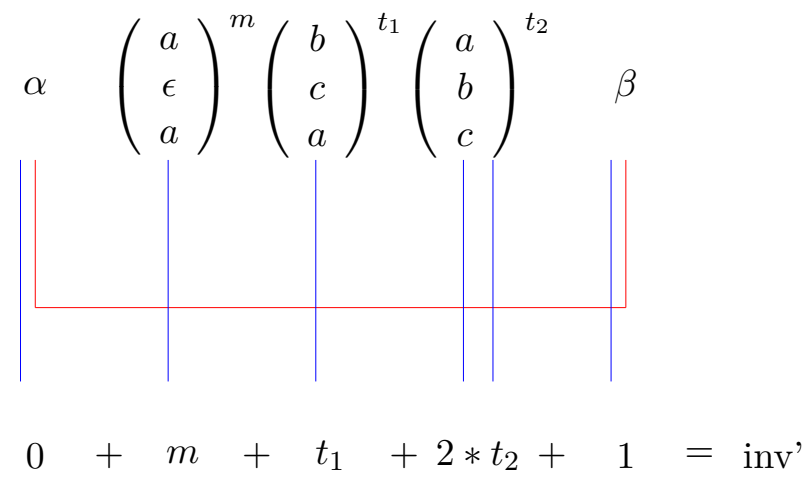

Corollary 43.

$$
\left.\sum a^{u} b^{v} c^{w} q^{m}=\sum a^{u} b^{v} c^{w} q_{2}^{\left(t_{1}-t_{2}+1\right.}\right) q^{i n v_{1}+i n v_{2}+i n v_{3}+n_{1}-n_{2}}=\sum a^{u} b^{v} c^{w} q^{\left(\begin{array}{c}
(+1 \\
2
\end{array}\right)} q^{i n v_{1}+i n v_{2}+i n v_{3}}
$$

\section{Conclusion}

As described in subsection 1.3.2, we computed first with a combinatorial approach Al-Salam-Carlitz moments using a sign reversing involution directly on the Motzkin paths. It was possible to adapt this proof for Askey-Wilson moments for the case $c=d=0$ giving an alternative combinatorial proof of what has been done by Stanton and Kim in [10] with doubly striped skew shapes. The same proof has also been adapted in order to define a sign reversing involution for the case $d=0$ of Askey-Wilson polynomials; giving a more complicated set of fixpoints that required to define another bijection. In order to pursue this work the natural next steps would be to generalize first to the most general Askey-Wilson moments (no restriction on $a, b, c, d)$ and then to other families of orthogonal polynomials. Note that the most general case is different because it is no longer a polynomial in $a, b, c$ and $d$.

\section{Acknowledgments and fundings}

I thank Dr. Jang Soo Kim for his collaboration and ideas, as well as his general support when I was in South Korea, where this work was made. We also thank the jury of Parisian Master of Research in Computer Science for its proof reading. This research was funded by the ENS Cachan, and partially supported by the Sungkyunkwan university. Since September 2016, my research has been fully supported by EPFL Doctoral School. 


\section{Contents}

1 Context and goal $\quad 2$

1.1 Notation and Definition . . . . . . . . . . . . . . . . . . . . . 2

1.2 Three combinatorial approaches . . . . . . . . . . . . . . . . . . . . . . . . . . . . .

1.2.1 First combinatorial approach: Motzkin paths . . . . . . . . . . . . . . 4

1.2.2 Second combinatorial approach: staircase tableaux . . . . . . . . . . . . . 5

1.2.3 Third combinatorial approach: $q$-Hermite polynomials . . . . . . . . . . 5

1.3 Goal of this article . . . . . . . . . . . . . . . . . . . . . 6

1.3.1 Presentation of the sign reversing involution method: the proof of theorem 3 . . 6

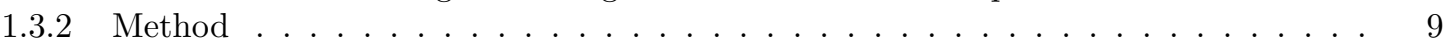

$2 \quad \mathbf{1 0}$

2.1 Definitions and intuition . . . . . . . . . . . . . . . . . . . . . . . . 10

2.2 Bijection with paired words . . . . . . . . . . . . . . . . . . . 11

2.3 Cancellation . . . . . . . . . . . . . . . . . . . . . . . . 11

2.4 Maximal paired words and fixpoints . . . . . . . . . . . . . . . . . . . 13

2.5 Theorem 3 is a generalization of the formula for Al-Salam-Carlitz moments . . . . . . . . 14

3 Askey-Wilson polynomials, case $c \neq 0 \quad 15$

3.1 Definitions . . . . . . . . . . . . . . . . . . . . . . . . 15

3.2 Bijection between words and Motzkin paths . . . . . . . . . . . . . . . . 17

3.3 Involution and cancellation . . . . . . . . . . . . . . . . . . . . 18

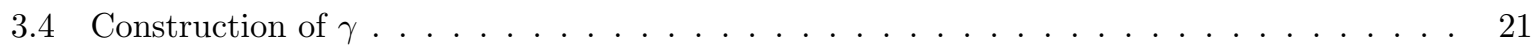

3.5 Concluding the proof . . . . . . . . . . . . . . . . . . . . . . 24

4 Proof of Lemma 35 with crossings $\quad 24$

5 Conclusion $\quad 27$

A Some cases for Kim and Stanton involution $\quad 30$

B Involution for Al-Salam Carlitz polynomials, example 30

C Mapping from words of $W_{a, b, c, a b c,-1}\left(u-t_{2}, v-t_{2}, w-t_{2}, t_{2}, t_{1}\right)$
to $W_{a, b, c, a b c,-1}\left(u-t_{2}, v-t_{2}, w-t_{2}, t_{2}, 2 t_{1}\right)$

D Definition of $f$

$\begin{array}{llr}\text { E Proof of Lemma } 10 & 34\end{array}$

F Proof of Lemma $34 \quad 35$

G Complete proof of Lemma $35 \quad 36$ 


\section{References}

[1] WA. Al-Salam and L. Carlitz, Some orthogonal q-polynomials. Mathematische Nachrichten, 30(12):47-61 1965 .

[2] R. Askey and J. A. Wilson, Some basic hypergeometric orthogonal polynomials that generalize Jacobi polynomials. American Mathematical Soc., 319, 1985.

[3] S. Corteel, R. Stanley, D. Stanton and L. Williams, Formulae for Askey-Wilson moments and enumeration of staircase tableaux. Transactions of the American Mathematical Society, 364(11):6009-6037, 2012.

[4] S. Corteel, L. K. Williams and others. Tableaux combinatorics for the asymmetric exclusion process and Askey-Wilson polynomials. Duke Mathematical Journal, 159(3):385-415, 2011.

[5] G. Gasper and M. Rahman. Basic hypergeometric series. Cambridge university press, 96, 2004.

[6] M. Ismail and D. Stanton. q-Taylor theorems, polynomial expansions, and interpolation of entire functions. Journal of Approximation Theory, 123(1):125-146, 2003.

[7] M. Ismail, D. Stanton and G. Viennot. The combinatorics of q-hermite polynomials and the AskeyWilson integral. European Journal of Combinatorics, 8(4):379-392, 1987.

[8] M.Jusuat-Vergés and M. Rubey Crossings, Motzkin paths and moments. Discrete mathematics, 311(18-19):2064-2078, 2011.

[9] D. Kim. On combinatorics of Al-Salam Carlitz polynomials. European Journal of Combinatorics, 18(3):295-302, 1997.

[10] J. Soo Kim, D. Stanton Moments of Askey-Wilson polynomials. Journal of Combinatorial Theory, Series A, 125:113-145, 2014.

[11] J. Soo. Kim and D. Stanton Bootstrapping and Askey-Wilson polynomials. Journal of Mathematical Analysis and Applications, 421(1):501-520, 2015.

[12] J-G. Penaud. Une preuve bijective d'une formule de Touchard-Riordan. Discrete Mathematics, 139(1-3):347-360, 1995.

[13] G. Viennot Une théorie combinatoire des polynômes orthogonaux généraux. Departement de Mathématiques et d'Informatique, Université du Québec à Montréal, 1983.

[14] G. Viennot A combinatorial theory for general orthogonal polynomials with extensions and applications Polynômes Orthogonaux et Applications, 139-157, 1985.

[15] G. Viennot Problemes combinatoires posés par la physique statistique. Séminaire Bourbaki, 26:225246, 1985 . 


\section{A Some cases for Kim and Stanton involution}
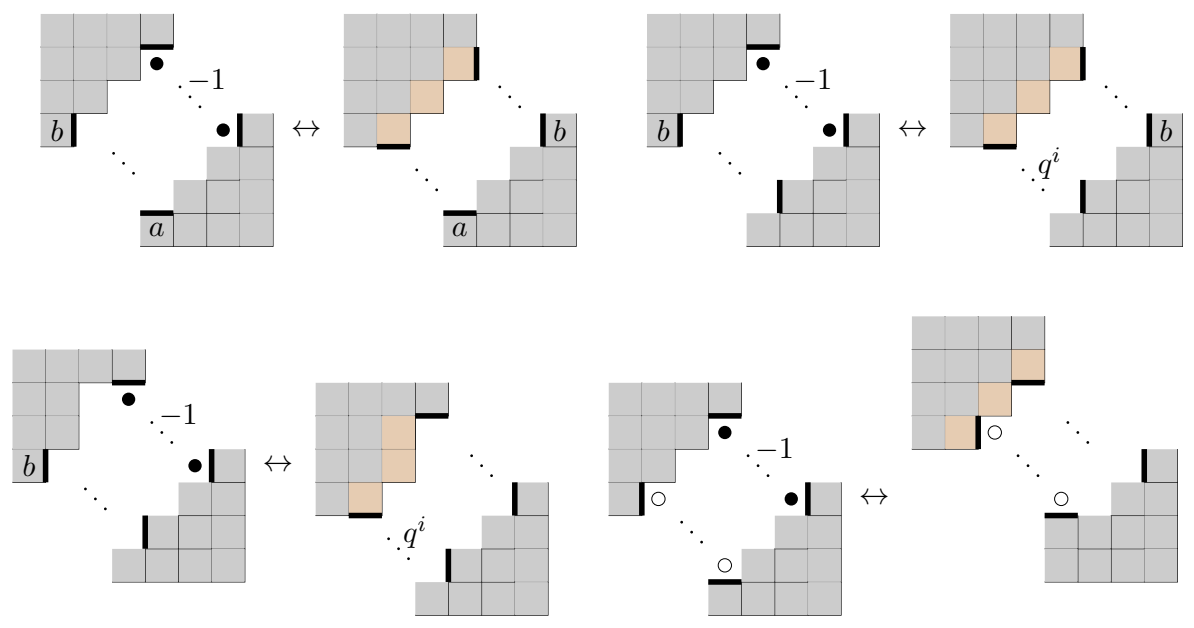

Figure 21: Possible actions of $\phi$

\section{B Involution for Al-Salam Carlitz polynomials, example}

$$
\left\{\begin{array}{cc}
-, & / \\
q^{i} & a q^{i}
\end{array}\right\} \quad\left\{\begin{array}{ll}
\searrow & - \\
q^{i} & \overline{a q^{i}}
\end{array}\right\} \quad\left\{\begin{array}{c}
\searrow \\
-1
\end{array}\right\}
$$

Figure 22: Reminder

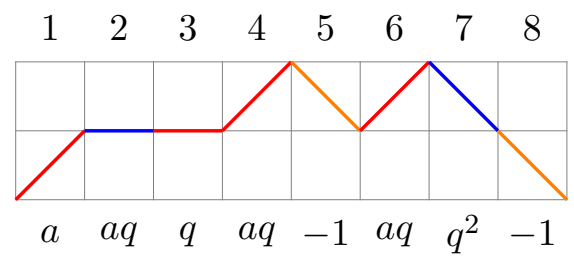

Figure 23: $\mathrm{P}$

In the following, the function $f$ will be the function defined in the figure 11 .

Let us recall how $\phi$ works in that case $\left(P \in E_{1}\right)$ :

- $i_{0}$ is the index of the first orange step, here 5 . Level $(5)=2$, thus 2 iterations.

- $i_{1}$ is the index of the last red step before $i_{0}$

- (i) Apply $f$ to $\left(P_{i_{1}}, P_{i_{1}+1}\right)$. This defines $P_{1}$. ( $1^{\text {st }}$ iteration $)$

- $i_{2}$ is the index of the last red step before $i_{1}$ (which is now a blue step)

- (ii) Apply $f$ to $\left(P_{i_{2}}, P_{i_{2}+1}\right)$. This defines $P_{2}=\phi(P)$. ( $2^{\text {nd }}$ iteration) 
(i)

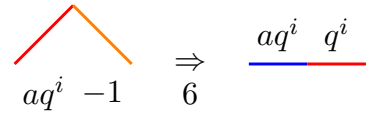

(ii) $\stackrel{q^{i} a q^{i}}{\underset{2}{\Rightarrow}} q^{i} a q^{i-1}$

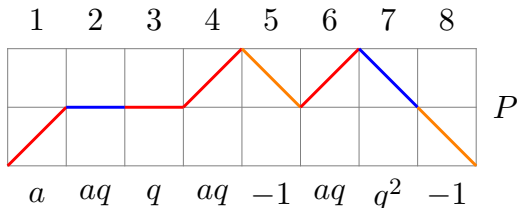

$\Downarrow(i)$

$\begin{array}{llllllll}1 & 2 & 3 & 4 & 5 & 6 & 7 & 8\end{array}$

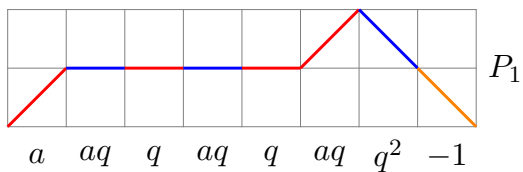

$\Downarrow(i i)$

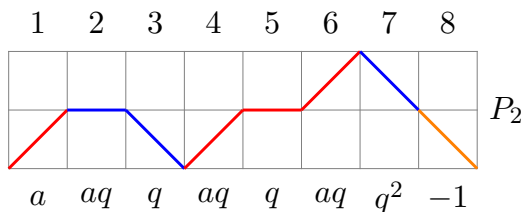

$w\left(p_{2}\right)=-a^{4} q^{7}=-w(P)$

\section{Mapping from words of $W_{a, b, c, a b c,-1}\left(u-t_{2}, v-t_{2}, w-t_{2}, t_{2}, t_{1}\right)$ to $W_{a, b, c, a b c,-1}\left(u-t_{2}, v-t_{2}, w-t_{2}, t_{2}, 2 t_{1}\right)$}

In this section we define a mapping from $W_{a, b, c, a b c,-1}\left(u-t_{2}, v-t_{2}, w-t_{2}, t_{2}, t_{1}\right)$ to $W_{a, b, c, a b c,-1}\left(u-t_{2}, v-\right.$ $\left.t_{2}, w-t_{2}, t_{2}, 2 t_{1}\right)$, which corresponds to doubling the number of -1 in the word; however this has to be done bijectively, meaning that all words of $W_{a, b, c, a b c,-1}\left(u-t_{2}, v-t_{2}, w-t_{2}, t_{2}, t_{1}\right)$ should be possible and all of them should correspond to a unique label of a Motzkin path in $F_{a, b, c}$.

Remark 44. Words of $W_{a, b, c, a b c,-1}\left(u-t_{2}, v-t_{2}, w-t_{2}, t_{2}, t_{1}\right)$ are of length $u+v+w+\left(2 * t_{1}-t_{2}\right)$, words of $W_{a, b, c, a b c,-1}\left(u-t_{2}, v-t_{2}, w-t_{2}, t_{2}, 2 t_{1}\right)$ are of length $u+v+w+2 *\left(t_{1}-t_{2}\right)$.

We extend the existing mapping defined in [10] for the case $c \neq 0$. This creates a word in $M_{a, b, c, a b c,-1}$ of length $u+v+w+t_{1}-2 * t_{2}$ from a Motzkin path in $F_{a, b, c}$. This mapping gives us a mapping from words of length $u+v+w+2 *\left(t_{1}-t_{2}\right)$ (the label of the Motzkin path) to words of length $u+v+w+2 * t_{1}-t_{2}$. Let $\delta$ be this mapping.

We finally define the function $\eta$ from triple of words to $M P_{a, b, c}^{u+v+w+2 t}$ as follows. Let $\left(w_{1}, w_{2}, w_{3}\right)$ be a triple of words, and $w=\gamma\left(w_{1}, w_{2}, w_{3}\right)$, then $\eta\left(w_{1}, w_{2}, w_{3}\right)$ is the unique $P$ Motzkin path such that $\delta(P)=w$. (In fact, $\left.\eta=\delta^{-1} \circ \gamma\right)$.

Let us call $\tilde{\eta}$ the function that gives from $\left(w_{1}, w_{2}, w_{3}\right)$ the corresponding Motzkin path. (Such that the label is the maximal pairing of $\eta(w))$

Lemma 45. $\tilde{\eta}$ is a bijection from

$\left[W_{a, b}(u, v+t) \times W_{b, c}(v, w+t) \times W_{c, a}(w, u+t)\right]$ to $F_{a, b, c}$.

Remark 46. There are four intermediate results to prove.

- $(i) \forall\left(w_{1}, w_{2}, w_{3}\right), \tilde{\eta}\left(w_{1}, w_{2}, w_{3}\right) \in F_{a, b, c}$

- (ii) For all word, there is only one maximal pairing (already proved)

- (iii) $\forall P \in F_{a, b, c}, \exists\left(w_{1}, w_{2}, w_{3}\right)$ such that $\tilde{\eta}=P$. 


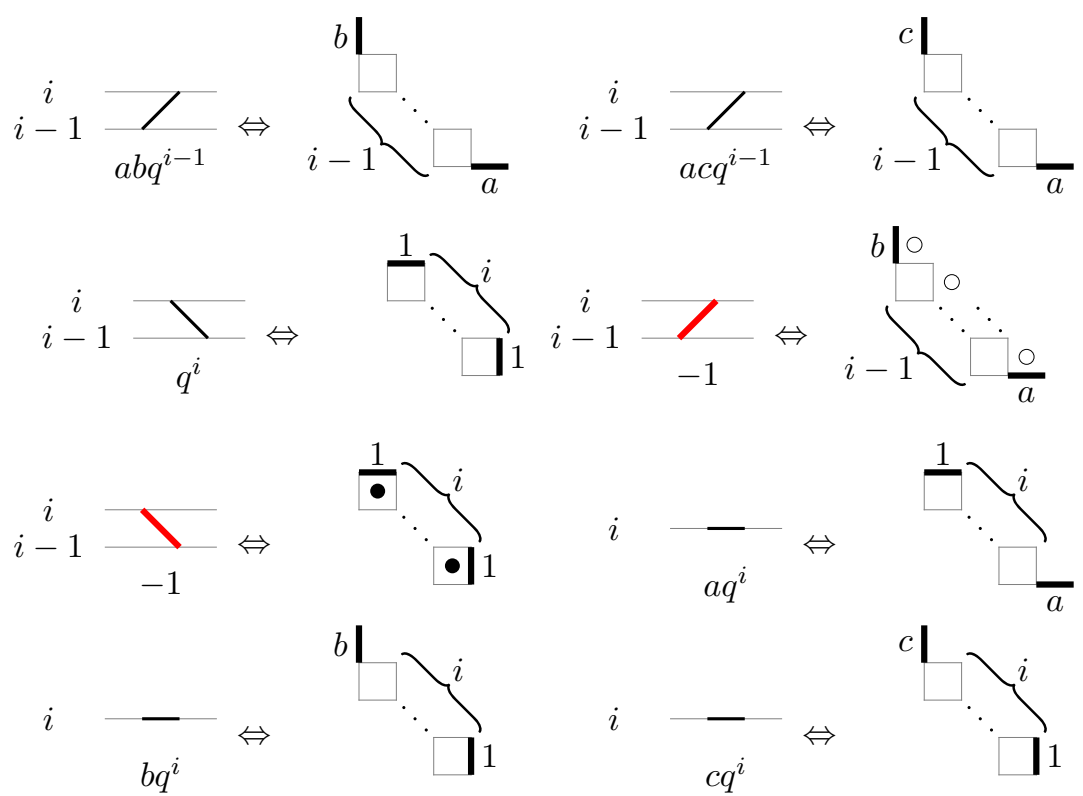

Figure 25: Extension of the map, part 1
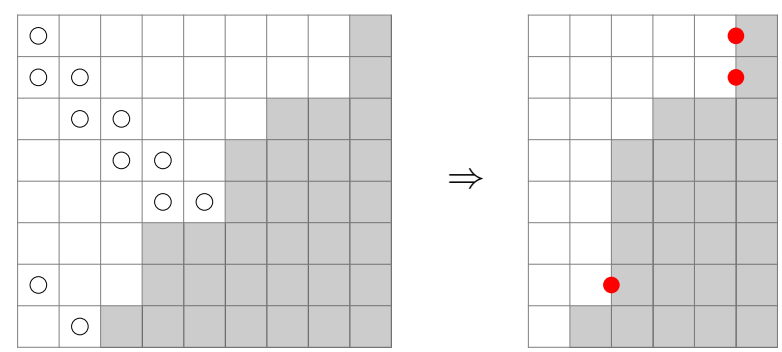

Figure 26: Extension of the map, part 2

- $(i v) \forall\left(w_{1}, w_{2}, w_{3}\right) \neq\left(w_{1}^{\prime}, w_{2}^{\prime}, w_{3}^{\prime}\right) \Rightarrow \tilde{\eta}\left(w_{1}, w_{2}, w_{3}\right) \neq \tilde{\eta}\left(w_{1}^{\prime}, w_{2}^{\prime}, w_{3}^{\prime}\right)$

Proof. (1) To prove (i) and (iii), we will prove later that a Motzkin path $P$ is in $F_{a, b, c}$ iff its corresponding paired word $(w, F)$ is maximal. This will thus prove $(i)$, and since $\gamma$ is a bijection, $\gamma^{-1}(w)$ is a triple $\left(w_{1}, w_{2}, w_{3}\right)$ such that $\tilde{\eta}\left(w_{1}, w_{2}, w_{3}\right)$ is the Motzkin path corresponding to the maximal pairing of $w$. If $(w, F)$ is maximal, since the maximal pairing is unique, $\eta\left(w_{1}, w_{2}, w_{3}\right)=(w, F)$ which proves (iii). (2) (iv) comes from the fact that $\gamma$ is a bijection thus injective, and the mapping previously from path to words is also injective, so $\eta$ is injective, thus $\tilde{\eta}$ is injective. 


\section{Definition of $f$}

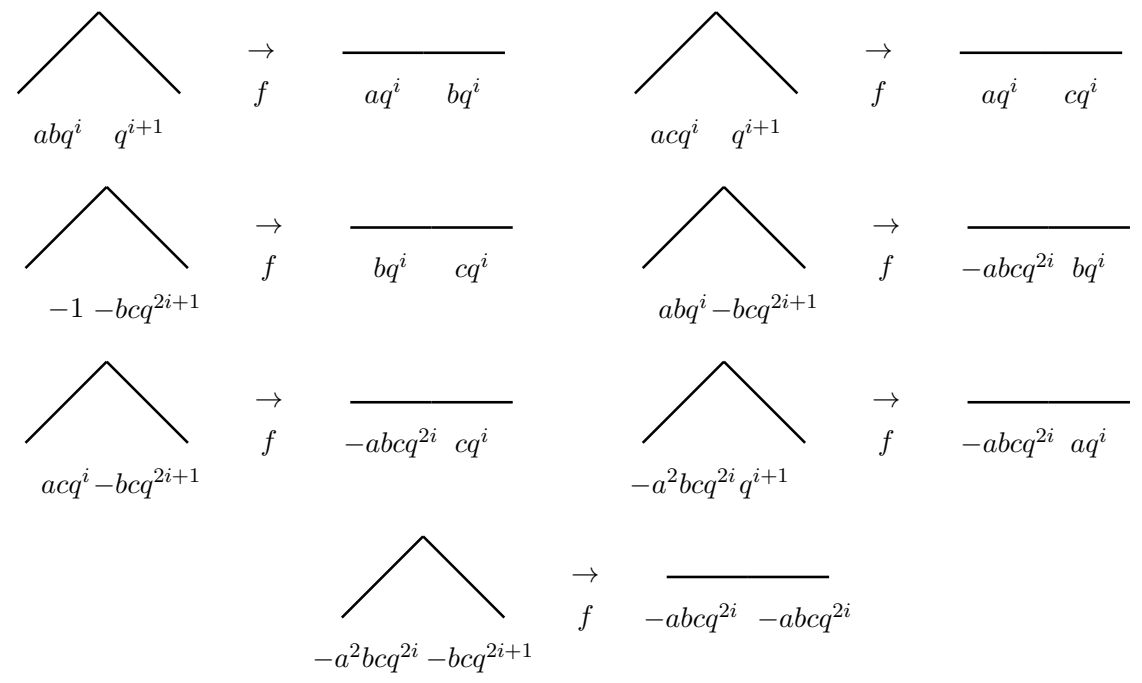

Figure 27: Definition of $f$ : Case $(i)$
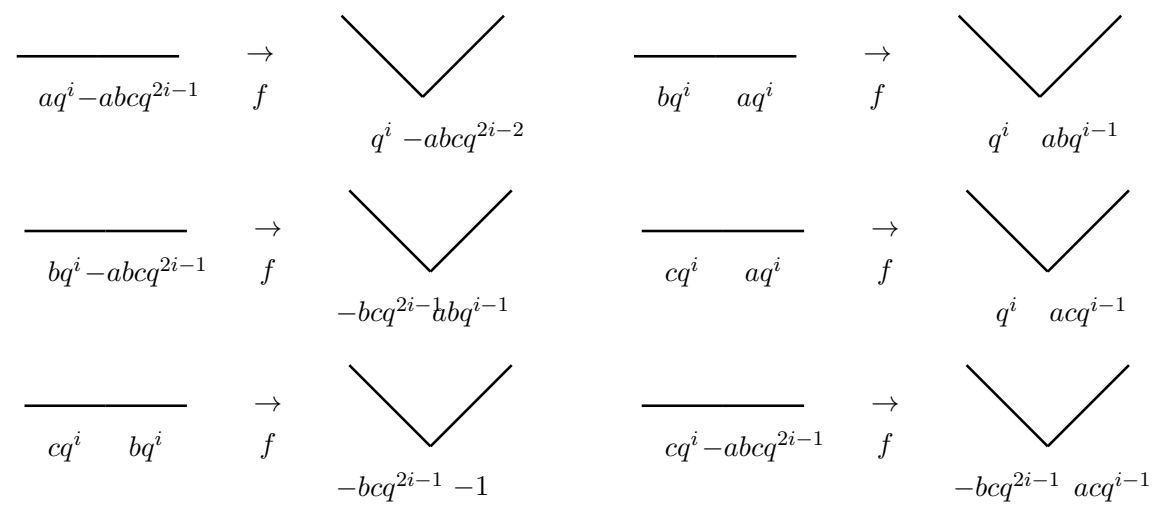

Figure 28: Definition of $f$ : Case $(i i)$ 


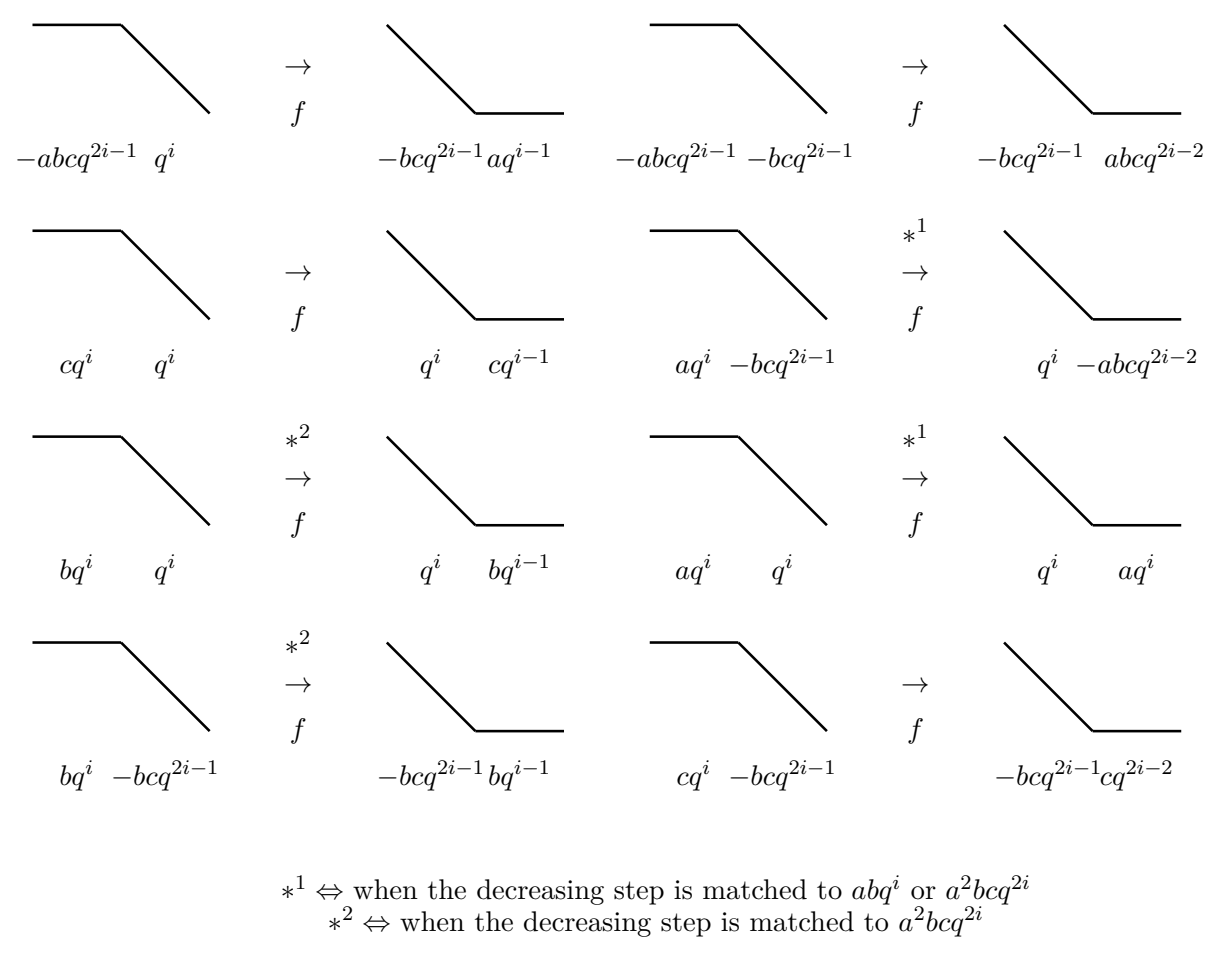

Figure 29: Definition of $f$ : Case $($ iii $)$

Remark 47. The star conditions are necessary because the decreasing step alone does not provide enough information to know if the corresponding letter $\alpha$ in the word is smaller than $\beta$. Defining the $f$ function directly on paired words would have prevented this issue.

\section{E Proof of Lemma 10}

Definition 48. In order to prove this result, we introduce a new mapping $g: \operatorname{Mot}_{n} \rightarrow\{A, B, C\}^{n}$ defined as the following.

- For $s$ possible step,

$$
\begin{aligned}
& -g(s)=A \Leftrightarrow s \in A \\
& -g(s)=B \Leftrightarrow s \in B \\
& -g(s)=C \Leftrightarrow s \in C
\end{aligned}
$$

- $g$ is a morphism.

Proof. We will prove the Lemma 10 by induction using the following property : after $k$ iterations,

$$
\text { \#Inversions } s_{i_{0}} \geqslant i-k
$$

where Inversions $_{i_{0}}=\left\{(i, j) \mid g(w)_{i}<g(w)_{j} \& i<j \leqslant i_{0}\right\}$ for the ordering $A<B$ and $A<C$.

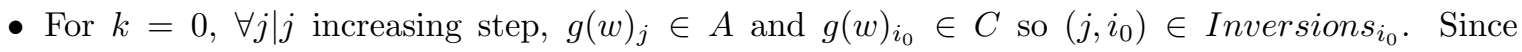
level $\left(i_{0}\right)=i$, \#Inversions $i_{0} \geqslant i$.

- For $k \geqslant 1$, Inversions $i_{0}>0$ so we can pick two consecutive indexes $\left(j_{1}, j_{2}\right)$ as given in the definition of $f$, such that $g\left(w\left(j_{1}\right)\right)<g\left(w\left(j_{2}\right)\right)$. With $\left(s_{1}, s_{2}\right)=f\left(g\left(w\left(j_{1}\right)\right), g\left(w\left(j_{2}\right)\right)\right), s_{1}>s_{2}$ so Inversions $_{i_{0}}^{\prime}=$ Inversion $_{i_{0}}-1=i-(k+1)$ which leads to the induction assumption at rank $k+1$. 


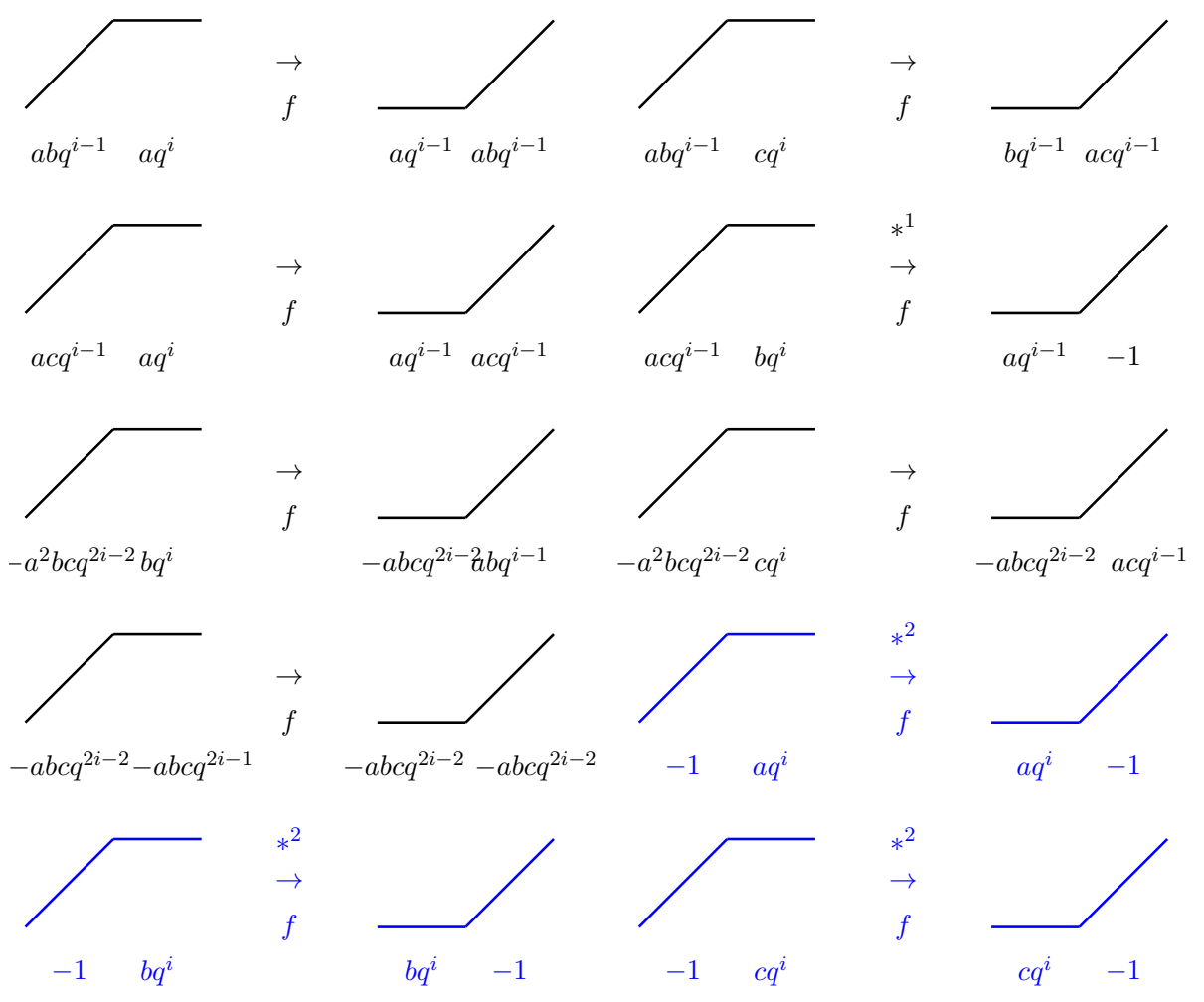

$*^{1} \Leftrightarrow$ The matching decreasing step has to be $q^{i}$ and becomes $-b c q^{2 i-1}$

The $*^{2}$ cases generalize the shifting of white dots on the right in the article [?] for the case $c=0$.

Figure 30: Definition of $f$ : Case $(i v)$

\section{F Proof of Lemma 34}

Proof. By induction on the word w. (In the following, $\epsilon$ stands for the empty word)

- If $w=\epsilon$, then $\gamma(w)=(\epsilon, \epsilon, \epsilon)$.

- If $w^{\prime}=w \cdot a$ then $\gamma\left(w^{\prime}\right)=\left(w_{1}, w_{2}, w_{3}\right) \cdot(a, \epsilon, a) u^{\prime}=u+1, v^{\prime}=v, w^{\prime}=w, t^{\prime}=t$.

$$
\begin{gathered}
\#_{a}\left(w_{1}^{\prime}\right)=\#_{a}\left(w_{1}\right)+1=u^{\prime} \quad \#_{b}\left(w_{1}^{\prime}\right)=v^{\prime}+t^{\prime} \\
\#_{b}\left(w_{2}^{\prime}\right)=\#_{b}\left(w_{2}\right)=v^{\prime} \quad \#_{c}\left(w_{2}^{\prime}\right)=w^{\prime}+t^{\prime} \\
\#_{c}\left(w_{1}^{\prime}\right)=\#_{c}\left(w_{3}\right)=w^{\prime} \quad \#_{a}\left(w_{3}^{\prime}\right)=\# a\left(w_{3}\right)+1=u^{\prime}+t^{\prime}
\end{gathered}
$$

- If $w^{\prime}=w . b$ then $\gamma\left(w^{\prime}\right)=\left(w_{1}, w_{2}, w_{3}\right)$. $(b, b, \epsilon) u^{\prime}=u, v^{\prime}=v+1, w^{\prime}=w, t^{\prime}=t$.

$$
\begin{gathered}
\#_{a}\left(w_{1}^{\prime}\right)=\#_{a}\left(w_{1}\right)=u^{\prime} \quad \#_{b}\left(w_{1}^{\prime}\right)=\#_{b}\left(w_{1}\right)+1=v^{\prime}+t^{\prime} \\
\#_{b}\left(w_{2}^{\prime}\right)=\#_{b}\left(w_{2}\right)+1=v^{\prime} \quad \#_{c}\left(w_{2}^{\prime}\right)=w^{\prime}+t^{\prime} \\
\#_{c}\left(w_{1}^{\prime}\right)=\#_{c}\left(w_{3}\right)=w^{\prime} \quad \#_{a}\left(w_{3}^{\prime}\right)=u^{\prime}+t^{\prime}
\end{gathered}
$$


- If $w^{\prime}=w . c$ then $\gamma\left(w^{\prime}\right)=\left(w_{1}, w_{2}, w_{3}\right)$. $(\epsilon, c, c) u^{\prime}=u, v^{\prime}=v, w^{\prime}=w+1, t^{\prime}=t$.

$$
\begin{gathered}
\#_{a}\left(w_{1}^{\prime}\right)=\#_{a}\left(w_{1}\right)=u^{\prime} \quad \#_{b}\left(w_{1}^{\prime}\right)=v^{\prime}+t^{\prime} \\
\#_{b}\left(w_{2}^{\prime}\right)=\#_{b}\left(w_{2}\right)=v^{\prime} \quad \#_{c}\left(w_{2}^{\prime}\right)=\#_{c}\left(w_{2}\right)+1=w^{\prime}+t^{\prime} \\
\#_{c}\left(w_{1}^{\prime}\right)=\#_{c}\left(w_{3}\right)+1=w^{\prime} \quad \#_{a}\left(w_{3}^{\prime}\right)=u^{\prime}+t^{\prime}
\end{gathered}
$$

- If $w^{\prime}=w \cdot(a b c)$ then $\gamma\left(w^{\prime}\right)=\left(w_{1}, w_{2}, w_{3}\right) \cdot(a, b, c) u^{\prime}=u+1, v^{\prime}=v+1, w^{\prime}=w+1, t^{\prime}=t-1$.

$$
\begin{aligned}
& \#_{a}\left(w_{1}^{\prime}\right)=\#_{a}\left(w_{1}\right)+1=u^{\prime} \quad \#_{b}\left(w_{1}^{\prime}\right)=\#_{b}\left(w_{1}\right)=v+1+t-1=v^{\prime}+t^{\prime} \\
& \#_{b}\left(w_{2}^{\prime}\right)=\#_{b}\left(w_{2}\right)+1=v^{\prime} \quad \#_{c}\left(w_{2}^{\prime}\right)=\#_{c}\left(w_{2}\right)=w+1+t-1=w^{\prime}+t^{\prime} \\
& \#_{c}\left(w_{1}^{\prime}\right)=\#_{c}\left(w_{3}\right)+1=w^{\prime} \quad \#_{a}\left(w_{3}^{\prime}\right)=\#_{a}\left(w_{3}\right)=u+1+t-1=u^{\prime}+t^{\prime}
\end{aligned}
$$

- If $w^{\prime}=w \cdot(-1)$ then $\gamma\left(w^{\prime}\right)=\left(w_{1}, w_{2}, w_{3}\right)$. $(b, c, a) u^{\prime}=u, v^{\prime}=v, w^{\prime}=w, t^{\prime}=t+1$.

$$
\begin{aligned}
& \#_{a}\left(w_{1}^{\prime}\right)=\#_{a}\left(w_{1}\right)=u^{\prime} \quad \#_{b}\left(w_{1}^{\prime}\right)=\#_{b}\left(w_{1}\right)+1=v+t+1=v^{\prime}+t^{\prime} \\
& \#_{b}\left(w_{2}^{\prime}\right)=\#_{b}\left(w_{2}\right)=v^{\prime} \quad \#_{c}\left(w_{2}^{\prime}\right)=\#_{c}\left(w_{2}\right)+1=w+t+1=w^{\prime}+t^{\prime} \\
& \#_{c}\left(w_{1}^{\prime}\right)=\#_{c}\left(w_{3}\right)=w^{\prime} \quad \#_{a}\left(w_{3}^{\prime}\right)=\#_{a}\left(w_{3}\right)+1=u+t+1=u^{\prime}+t^{\prime}
\end{aligned}
$$

\section{G Complete proof of Lemma 35}

Lemma 49. For $P \in F_{a, b, c}$ such that $w(p)=a^{u} b^{v} c^{w} q^{m}$, and $\gamma(P)=\left(w_{1}, w_{2}, w_{3}\right)$ then $m=\left(\begin{array}{c}t_{1}-t_{2}+1 \\ 2\end{array}\right)+i n v_{1}\left(w_{1}\right)+i n v_{2}\left(w_{2}\right)+i n v_{3}\left(w_{3}\right)+n_{1}-n_{2}$. Where inv $v_{1}(w)=$ $\#\{(i, j) \mid w(i)=a \& w(j)=b\}, \operatorname{inv}_{2}(w)=\#\{(i, j) \mid w(i)=b \& w(j)=c\}, \operatorname{inv}_{3}(w)=\#\{(i, j) \mid w(i)=$ $c \& w(j)=a\}$.

Proof. First, let us make some remarks which will be useful for the proof of the lemma. For convenience, let us define $i n v=\left(i n v_{1}+i n v_{2}+i n v_{3}\right)+n_{1}-n_{2}$ (which is not the number of inversion in the resulting word).

- (i) if $\alpha<\beta$ for the order $a<b<c$, then $\operatorname{inv}(\alpha \beta)=1$

- (ii) if $\alpha>\beta$ for the order $a<b<c$, then $i n v(\alpha \beta)=0$

- (iii) $\forall \alpha \in\{a, b, c, a b c,-1\}, \operatorname{inv}(\alpha \alpha)=0$

- (iv) if $\alpha<\beta$ for the order $a<b<c$, then $\operatorname{inv}\left(\alpha\left(\begin{array}{l}a \\ b \\ c\end{array}\right) \beta\right)=3$

- (v) $\forall \alpha \in\{a, b\}, \operatorname{inv}\left(\alpha\left(\begin{array}{c}b \\ c \\ a\end{array}\right)\right)=1$

In the following, $\left(\begin{array}{l}w_{1} \\ w_{2} \\ w_{3}\end{array}\right)$ will stand for an element of

$$
\left[W_{a, b}(u, v+t) \times W_{b, c}(v, w+t) \times W_{c, a}(w, u+t)\right],
$$

$w$ the corresponding word in $W_{a, b, c, a b c,-1}\left(u-t_{2}, v-t_{2}, w-t_{2}, t_{2}, t_{1}\right)$ and $\delta(w)$ the corresponding word in $W_{a, b, c, a b c,-1}\left(u-t_{2}, v-t_{2}, w-t_{2}, t_{2}, 2 t_{1}\right)$. By induction on the pairs, with the hypothesis: $\operatorname{inv}_{1}\left(w_{1}\right)+$ $\operatorname{inv}_{2}\left(w_{2}\right)+\operatorname{inv}_{3}\left(w_{3}\right)+n_{1}-n_{2}+\left(\begin{array}{c}t_{1}-t_{2}+1 \\ 2\end{array}\right)=($ number of $\operatorname{crossings})(\delta(w))$. 
- If $w$ does not contain any pair, according to the fact that the pairing should be maximal for the previously defined pairing rules, this means the number of inversion is also 0 , as the number of -1 . The number of $a b c$ is 0 or 1 , because if it is greater than 2, at least $2 a b c$ could be paired together.

- If $w^{\prime}=\alpha w \beta$, with $\alpha, \beta \in\{a, b, c\}$, where $w$ is a word with $m$ letters in $\{a, b, c\}, t_{1}$ in $\{-1\}$ and $t_{2}$ in $\{a b c\}$.

$$
\begin{aligned}
i n v^{\prime} & =i n v\left(\alpha\left(\begin{array}{l}
a \\
\epsilon \\
a
\end{array}\right)^{m}\left(\begin{array}{l}
b \\
c \\
a
\end{array}\right)^{t_{1}}\left(\begin{array}{l}
a \\
b \\
c
\end{array}\right)^{t_{2}} \beta\right)-i n v\left(\left(\begin{array}{l}
a \\
\epsilon \\
a
\end{array}\right)^{m}\left(\begin{array}{l}
b \\
c \\
a
\end{array}\right)^{t_{1}}\left(\begin{array}{l}
a \\
b \\
c
\end{array}\right)^{t_{2}}\right) \\
& ==m+t_{1}+2 * t_{2}+1
\end{aligned}
$$

( $a$ and $b$ behaves the same according to (iv)). Also,

$$
\begin{aligned}
\text { crossings }^{\prime}=\text { crossings }\left(\alpha\left(\begin{array}{l}
a \\
\epsilon \\
a
\end{array}\right)^{m}\left(\begin{array}{l}
b \\
c \\
a
\end{array}\right)^{t_{1}}\left(\begin{array}{l}
a \\
b \\
c
\end{array}\right)^{t_{2}} \beta\right) & - \text { crossings }\left(\left(\begin{array}{l}
a \\
a \\
\epsilon
\end{array}\right)^{m}\left(\begin{array}{l}
b \\
c \\
a
\end{array}\right)^{t_{1}}\left(\begin{array}{l}
a \\
b \\
c
\end{array}\right)^{t_{2}}\right)
\end{aligned}
$$

Which is what we want, indeed:

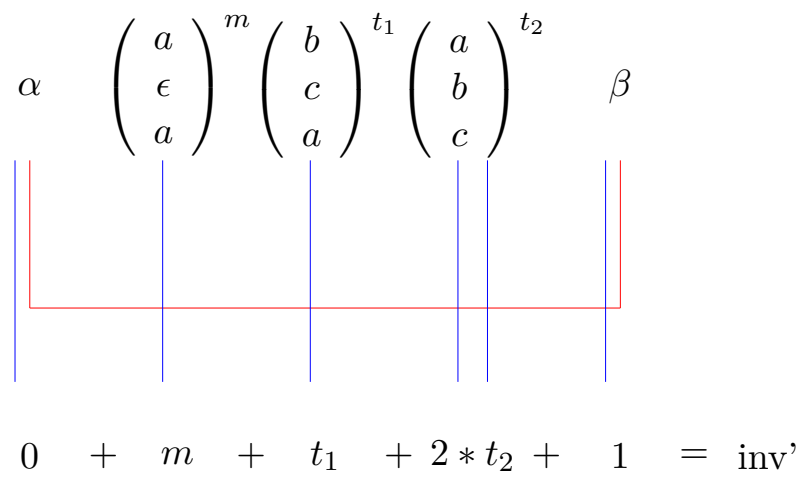

- If $\delta\left(w^{\prime}\right)=(-1) \delta(w)(-1)$, which means $w^{\prime}=w(-1)$ (plus some conditions), where $w$ is a word with $m$ letters in $\{a, b, c\}, t_{1}$ in $\{-1\}$ and $t_{2}$ in $\{a b c\}$.

$$
\begin{aligned}
\operatorname{inv} v^{\prime} & =i n v\left(\left(\begin{array}{l}
a \\
\epsilon \\
a
\end{array}\right)^{m}\left(\begin{array}{l}
b \\
c \\
a
\end{array}\right)^{t_{1}}\left(\begin{array}{l}
a \\
b \\
c
\end{array}\right)^{t_{2}}\left(\begin{array}{l}
b \\
c \\
a
\end{array}\right)\right)-i n v\left(\left(\begin{array}{l}
a \\
\epsilon \\
a
\end{array}\right)^{m}\left(\begin{array}{l}
b \\
c \\
a
\end{array}\right)^{t_{1}}\left(\begin{array}{l}
a \\
b \\
c
\end{array}\right)^{t_{2}}\right) \\
& =m+3 * t_{2}
\end{aligned}
$$

Also,

$$
\text { crossings }^{\prime}=\text { crossings }\left(\left(\begin{array}{c}
a \\
\epsilon \\
a
\end{array}\right)^{m}\left(\begin{array}{l}
b \\
c \\
a
\end{array}\right)^{t_{1}}\left(\begin{array}{l}
a \\
b \\
c
\end{array}\right)^{t_{2}}\left(\begin{array}{l}
b \\
c \\
a
\end{array}\right)\right)
$$




$$
\begin{aligned}
& (-1)\left(\begin{array}{c}
a \\
\epsilon \\
a
\end{array}\right)^{m}\left(\begin{array}{l}
b \\
c \\
a
\end{array}\right)^{t_{1}}\left(\begin{array}{l}
a \\
b \\
c
\end{array}\right)^{t_{2}}(-1) \\
& || \mid \\
& 0+m+t_{1}+2 * t_{2}+1 \\
& \text { - crossings }\left(\left(\begin{array}{c}
a \\
\epsilon \\
a
\end{array}\right)^{m}\left(\begin{array}{c}
b \\
c \\
a
\end{array}\right)^{t_{1}}\left(\begin{array}{c}
a \\
b \\
c
\end{array}\right)^{t_{2}}\right)
\end{aligned}
$$

So $i n v^{\prime}+\left(t_{1}-t_{2}\right)+1=$ crossings $^{\prime}$, which gives the expected result since

$$
\left(\begin{array}{c}
\left(t_{1}+1\right)-t_{2}+1 \\
2
\end{array}\right)=\left(\begin{array}{c}
t_{1}-t_{2}+1 \\
2
\end{array}\right)+\left(t_{1}-t_{2}\right)+1
$$

(arithmetic sum)

- If $\left(w^{\prime}\right)=(a b c) w \beta$, with $\beta \in\{a, b, c\}$, where $w$ is a word with $m$ letters in $\{a, b, c\}, t_{1}$ in $\{-1\}$ and $t_{2}$ in $\{a b c\}$.

$$
\begin{aligned}
i n v^{\prime} & =i n v\left(\left(\begin{array}{c}
a \\
b \\
c
\end{array}\right)\left(\begin{array}{c}
a \\
\epsilon \\
a
\end{array}\right)^{m}\left(\begin{array}{c}
b \\
c \\
a
\end{array}\right)^{t_{1}}\left(\begin{array}{l}
a \\
b \\
c
\end{array}\right)^{t_{2}}\left(\begin{array}{l}
b \\
b \\
\epsilon
\end{array}\right)\right)-i n v\left(\left(\begin{array}{l}
a \\
a \\
\epsilon
\end{array}\right)^{m}\left(\begin{array}{l}
b \\
c \\
a
\end{array}\right)^{t_{1}}\left(\begin{array}{l}
a \\
b \\
c
\end{array}\right)^{t_{2}}\right) \\
& =m+2 * t_{1}+t_{2}+1
\end{aligned}
$$

Also,

$$
\begin{array}{r}
\text { crossings }^{\prime}=\text { crossings }\left(\left(\begin{array}{l}
a \\
\epsilon \\
a
\end{array}\right)^{m}\left(\begin{array}{l}
b \\
c \\
a
\end{array}\right)^{t_{1}}\left(\begin{array}{l}
a \\
b \\
c
\end{array}\right)^{t_{2}}\left(\begin{array}{c}
b \\
c \\
a
\end{array}\right)\right) \\
- \text { crossings }\left(\left(\begin{array}{c}
a \\
\epsilon \\
a
\end{array}\right)^{m}\left(\begin{array}{c}
b \\
c \\
a
\end{array}\right)^{t_{1}}\left(\begin{array}{l}
a \\
b \\
c
\end{array}\right)^{t_{2}}\right)
\end{array}
$$

So $i n v^{\prime}+\left(t_{2}-t_{1}\right)=$ crossings $^{\prime}$, which gives the expected result since

$$
\left(\begin{array}{c}
t_{1}-\left(t_{2}+1\right)+1 \\
2
\end{array}\right)=\left(\begin{array}{c}
t_{1}-t_{2}+1 \\
2
\end{array}\right)+\left(t_{2}-t_{1}\right)
$$

(arithmetic sum)

- If $w^{\prime}=(a b c) w(a b c)$, where $w$ is a word with $m$ letters in $\{a, b, c\}, t_{1}$ in $\{-1\}$ and $t_{2}$ in $\{a b c\}$.

$$
i n v^{\prime}=i n v\left(\left(\begin{array}{c}
a \\
b \\
c
\end{array}\right)\left(\begin{array}{c}
a \\
\epsilon \\
a
\end{array}\right)^{m}\left(\begin{array}{c}
b \\
c \\
a
\end{array}\right)^{t_{1}}\left(\begin{array}{l}
a \\
b \\
c
\end{array}\right)^{t_{2}}\left(\begin{array}{l}
a \\
b \\
c
\end{array}\right)\right)-i n v\left(\left(\begin{array}{l}
a \\
a \\
\epsilon
\end{array}\right)^{m}\left(\begin{array}{l}
b \\
c \\
a
\end{array}\right)^{t_{1}}\left(\begin{array}{l}
a \\
b \\
c
\end{array}\right)^{t_{2}}\right)
$$




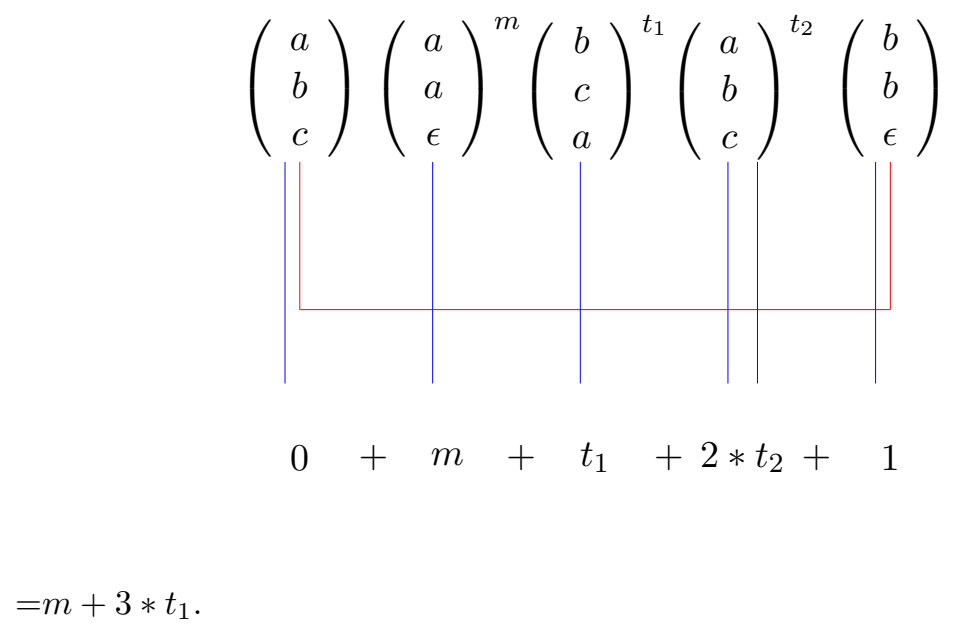

Also,

$$
\begin{aligned}
& \text { crossings }^{\prime}=\text { crossings }\left(\left(\begin{array}{l}
a \\
b \\
c
\end{array}\right)^{m}\left(\begin{array}{l}
b \\
c \\
a
\end{array}\right)^{t_{1}}\left(\begin{array}{l}
a \\
b \\
c
\end{array}\right)^{t_{2}}\left(\begin{array}{l}
a \\
b \\
c
\end{array}\right)\right) \\
& - \text { crossings }\left(\left(\begin{array}{c}
a \\
\epsilon \\
a
\end{array}\right)^{m}\left(\begin{array}{l}
b \\
c \\
a
\end{array}\right)^{t_{1}}\left(\begin{array}{l}
a \\
b \\
c
\end{array}\right)^{t_{2}}\right) \\
& \left(\begin{array}{l}
a \\
b \\
c
\end{array}\right)\left(\begin{array}{l}
a \\
a \\
\epsilon
\end{array}\right)^{m}\left(\begin{array}{l}
b \\
c \\
a
\end{array}\right)^{t_{1}}\left(\begin{array}{l}
a \\
b \\
c
\end{array}\right)^{t_{2}}\left(\begin{array}{l}
a \\
b \\
c
\end{array}\right) \\
& \mid \\
& 0+m+t_{1}+2 * t_{2}+1
\end{aligned}
$$

So $i n v^{\prime}-\left(t_{1}-t_{2}\right)-\left(t_{1}-t_{2}-1\right)=$ crossings $^{\prime}$, which gives the expected result since

$$
\left(\begin{array}{c}
t_{1}-\left(t_{2}+2\right)+1 \\
2
\end{array}\right)=\left(\begin{array}{c}
t_{1}-t_{2}+1 \\
2
\end{array}\right)-\left(t_{1}-t_{2}\right)-\left(t_{1}-t_{2}-1\right)
$$

(arithmetic sum) 